\title{
Co-administration of combretastatin A4 nanoparticles and anti-PD-L1 for synergistic therapy of hepatocellular carcinoma
}

\author{
Bonan Zhao ${ }^{1}$, Zhipeng Dong ${ }^{1}$, Weixing Liu ${ }^{1}$, Fangning Lou', Qiyan Wang ${ }^{1}$, Hao Hong ${ }^{2 *}$ and Yue Wang ${ }^{1 *}$
}

\begin{abstract}
Background: According to data estimated by the WHO, primary liver cancer is currently the fourth most common malignant tumor and the second leading cause of death around the world. Hepatocellular carcinoma (HCC) is one of the most common primary liver malignancies, so effective therapy is highly desired for HCC.

Results: In this study, the use of poly(L-Aspartic acid)-poly(ethylene glycol)/combretastatin A4 (CA4-NPs) was aimed to significantly disrupt new blood vessels in tumor tissues for targeted hepatic tumor therapy. Here, PEG-b-PAsp-gCA4 showed significantly prolonged retention in plasma and tumor tissue. Most importantly, CA4-NPs were mainly distributed at the tumor site because of the triple target effects-enhanced permeability and retention (EPR) effect, acid-sensitive ( $\mathrm{pH}=5.5$ ) effect to the tumor microenvironment (TME), and good selectivity of CA4 for central tumor blood vessel. Considering that CA4-NPs might induce severe hypoxic conditions resulting in high expression of HIF-1a in tumor tissues, which could induce the overexpression of PD-L1, herein we also used a programmed death-ligand 1 antibody (aPD-L1) to prevent immunosuppression. This way of complementary combination is able to achieve an ideal treatment effect in tumor site where CA4-NPs and APD-L1 could respond to the inner area and peripheral area, respectively. As a result, a significant decrease in tumor volume and weight was observed in the combination group of CA4-NPs plus aPD-L1 compared with CA4-NPs or APD-L1 monotherapy in subcutaneous Hepa 1-6 hepatic tumor models.
\end{abstract}

Conclusions: We presented a new idea that co-administration of CA4-NPs and aPD-L1 possessed notable anti-tumor efficacy for HCC treatment.

Keywords: Combretastatin A4(CA4), Nanoparticles, Anti-PD-L1(aPD-L1), Synergistic therapy

\section{Introduction}

Hepatocellular carcinoma (HCC) is the second leading cause of cancer-related death worldwide and more than half of the new cases and deaths occurred in Asia $[1,2]$. As it is challenging to diagnose HCC early, a principal

\footnotetext{
*Correspondence: haohong@nju.edu.cn; zwy_1115@126.com

${ }^{1}$ Key Laboratory of Biomedical Functional Materials, School of Sciences, China Pharmaceutical University, 639 Longmian Avenue, Nanjing 211198, China

2 School of Medicine, Nanjing University, 22 Hankou Road, Nanjing 210093, China
}

therapeutic challenge is the management of its highly malignant features and rapid progression. Due to the low rate of early diagnosis and rapid disease progression, the mortality rate of HCC remains high [3]. Surgery and liver transplantation are the best treatments for patients with liver cancer while the clinical treatment of advanced unresectable liver cancer remains a challenge. Therefore, for patients with unresectable hepatocellular carcinoma, transarterial chemoembolization (TACE) is recommended. However, TACE is a local treatment method, which is not effective in treating large tumors and advanced liver cancer [4-6]. The concurrent side 
effects also limit the application of TACE such as liver failure and gastrointestinal bleeding, etc. [7, 8]. But a large amount of clinical data showed that the use of traditional chemotherapy drugs to replace TACE did not bring satisfactory anti-tumor effects, so the development of new effective drugs against HCC has important clinical significance.

Vascular disrupting agents (VDAs) differ from traditional chemotherapy drugs in that they can not only eliminate the immature blood vessels in tumors preferentially but also modulate the tumor microenvironment (TME) selectively [9-11]. Combretastatin A4 phosphate (CA4P), as a representative VDA, can cause significant central tumor necrosis while leaving a thin layer of viable tumor cells at the tumor periphery $[12,13]$. CA4 itself has high selectivity for formed tumor blood vessels. Actually, a critical point in improving the therapeutic efficacy of CA4 further is to keep a constant concentration around the endothelial cells and enhance the action time on tubulin. Nanocarrier-based drug delivery systems provide an ideal platform to work out. Nanocarrierenwrapped or nanocarrier-conjugated drugs have the advantage of long-term circulation and high drug accumulation in the tumor region owing to the enhanced permeability and retention (EPR) effect. This system allows for gradual or temporary release of free active drugs from the nanocarriers in a controlled fashion and a constant drug concentration in the tumor tissue [14-16]. It is well known that poly(L-Aspartic)-polymeric-drug conjugates have consistently been used for drug development as PAsp is biocompatible, biodegradable, and easily modified to be a drug conjugate $[17,18]$. Similarly, the carboxyl group on the structure of Aspartic acid can be esterified with the hydroxyl group on the CA4 structure to form a nano-prodrug. As a result, the nano-drug delivery system constructed by chemical bonding is more stable than physical enwrapping and may be sensitive to tumor acidic environment [19].

Immune checkpoint blockade (ICB) that aims to reverse signals from the immunosuppressive TME is being driven as a primary treatment modality [20-22]. The efficacy of ICB-based cancer immunotherapy largely depends on the expression of PD-L1 in the tumor tissues and the recruitment of tumor-infiltrating lymphocytes (TILs) $[23,24]$. Owing to the high interstitial pressure inside the tumor center, the infiltration of $\mathrm{T}$ cells is hard to get inside the central area of tumor. As a result, they are easier to exert anti-tumor effect in the periphery [25]. Numerous related reports also show that the overexpression of HIF- $1 \alpha$ could induce the overexpression of PD-L1 in tumor tissues [26-29]. It is known that CA4 after delivered by nanoparticles in vivo could elicit central tumor necrosis and leaving normal tissues relatively impregnable, which exacerbate hypoxia in tumor tissue [30-33]. This phenomenon reveals that anti-PD-L1 should really be synergistic to inhibit tumor growth and reoccurrence in case of overexpression of PD-L1 during the treatment of CA4. Actually, as the landscape of antiPD-1/PD-L1 clinical trials moves towards combination strategies, which may be more efficacious, a recent report revealed that 253 drug target groups (excluding PD-1/ PD-L1) are being tested, which represents an increase of 129 new combined target groups in the past 3 years [34]. Hence, to design a combined treatment with aPD-L1 and CA4 for cancer is quite significant.

Therefore, in this study, nanoparticles of poly(L-Aspartic acid)-poly(ethylene glycol)/combretastatin A4 (CA4NPs) were prepared to improve the water solubility and prolong the half-life of CA4. CA4 itself has good selectivity for central tumor blood vessels and after being linked with PEG-b-PAsp by ester bond, CA4-NPs could offer significant advantages of EPR effect and acid-sensitive $(\mathrm{pH}=5.5)$ effect to TME. And a combination of CA4NPs plus aPD-L1 was tested in subcutaneous Hepa1-6 hepatic tumor models. The CA4-NPs effectively disrupted established tumor blood vessels, caused extensive tumor necrosis and inhibited tumor growth, but induced severe hypoxic conditions which resulted in the overexpression of PD-L1 in tumor tissues. Then co-administration of aPD-L1 can efficiently up-regulate the growth and proliferation of $\mathrm{T}$ cells and activate their attack and killing functions. As a result, this combination of treatment could achieve remarkable efficacy for HCC due to the regulation of tumor microenvironment (Scheme 1).

\section{Results and discussion}

\section{Preparation and characterization of PEG-b-PAsp-g-CA4}

In this study, CA4 was conjugated to PEG-b-PAsp using a one-step Yamaguchi reaction catalyzed by 2,4,6-trichlorobenzoyl chloride and DMAP [14]. The chemical structure of the formed PEG-b-PAsp and PEGb-PAsp-g-CA4 was confirmed by ${ }^{1} \mathrm{H}$ NMR (Fig. 1a). The peaks around 3.51 (d) $\mathrm{ppm}$ were attributable to the protons $\left(-\mathrm{CH}_{2} \mathrm{CH}_{2} \mathrm{O}\right)$ in the PEG chain. The peak around 12.5 (a), 8.06 (b), 4.9 (c), and 2.7 (e) ppm are attributable to the protons $(-\mathrm{COOH},-\mathrm{CONH}-,-\mathrm{COCHNH}-$, $\mathrm{CH}_{2} \mathrm{COOH}$ ) in the PAsp chain. Resonance peaks at 6.85 (f), $6.80(\mathrm{~g}), 6.70(\mathrm{~h}), 6.58(\mathrm{i})$ and 6.40 (j) 3.83 (k) 3.71 (l) ppm were attributed to the presence of CA4. Since the particle size, $\zeta$ potential, and stability were considered as significant factors that determine the fate of nanodrugs, the appearance and stability of PEG-b-PAsp and PEGb-PAsp-g-CA4 were characterized. The size of PEG-bPAsp was $102.1 \mathrm{~nm}(\mathrm{PDI}=0.252)$ and after being linked with CA4, the size changed to $153.5 \mathrm{~nm}(\mathrm{PDI}=0.184)$, which was consistent with the result of TEM respectively 


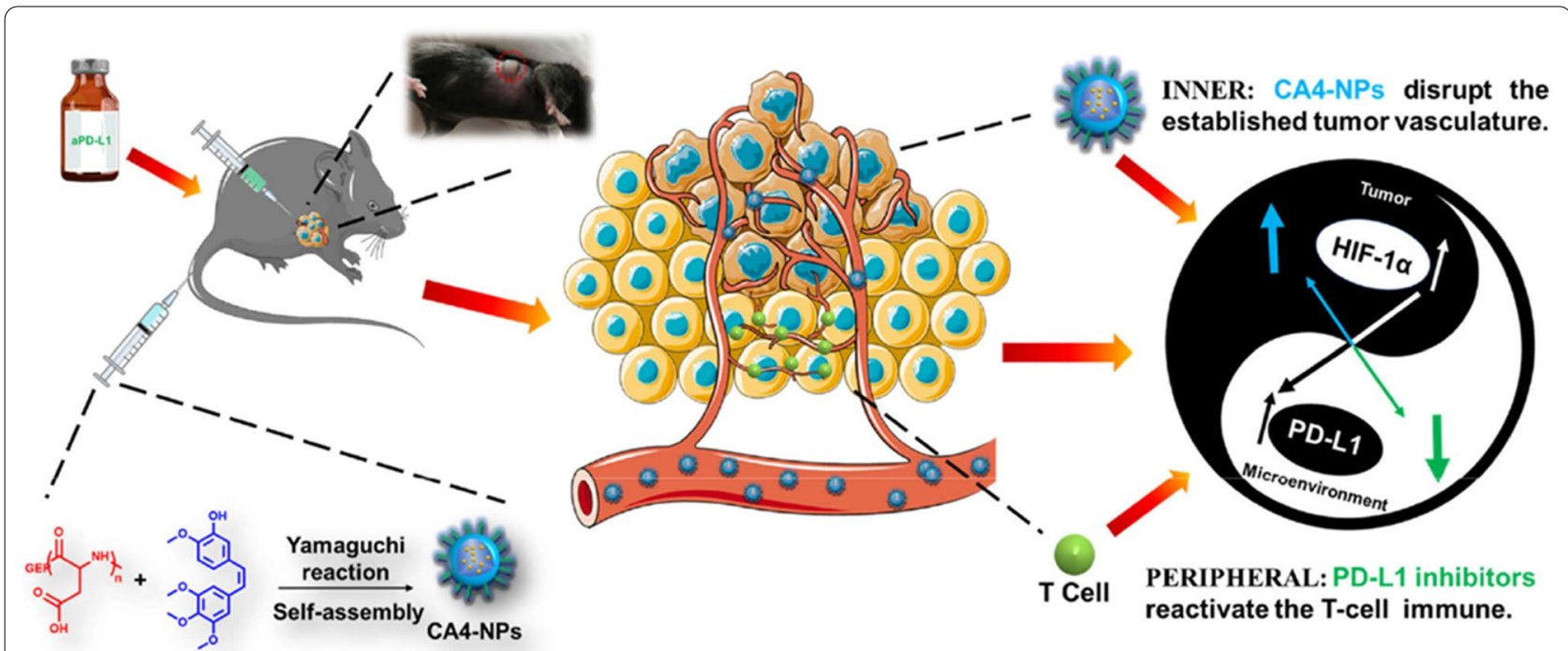

Scheme 1 CA4-NPs arrest the tumor by disrupting the established central tumor vasculature, which can induce severe hypoxic conditions and high expression of HIF-1a in tumor tissues. The hypoxic condition can increase the expression of PD-L1 in tumor microenvironment, so the PD-L1 inhibitors can release the 'immune brake' to reactivate the T-cell immune response to the tumor effect in the peripheral area

(Fig. 1b). Notably, the diameters of these NPs remained less than $200 \mathrm{~nm}$ after drug loading, which was highly suitable for passive tumor targeting for drug delivery by the EPR effect and not easy to be cleared due to too smaller particle size. The zeta potentials of the NPs, which were - 0.44 (PEG-b-PAsp) and $-27.56 \mathrm{mV}$ (PEGb-PAsp-g-CA4), were suitable for intravenous administration. The result of the relative molecular mass of polymer was determined by Gel Permeation Chromatography (GPC) in Fig. 1c. The peak time of PEG-b-PAspg-CA4 was earlier than PEG-b-PAsp, which meant that relative molecular mass of CA4-NPs was bigger and no detectable free $\mathrm{CA} 4$ was found in the conjugates. The CMC value for PEG-b-PAsp and PEG-b-PAsp-g-CA4 was measured to be $19.43 \mathrm{mg} \mathrm{L}{ }^{-1}$ and $13.44 \mathrm{mg} \mathrm{L}^{-1}$ respectively (Fig. 1d).

\section{In vitro drug release}

The CA4-NPs in pH 5.5 PBS buffer after $48 \mathrm{~h}$ were also detected by DLS, TEM, and GPC to mimic the drug release action in acidic tumor microenvironment. The diameter was about $132.7 \mathrm{~nm}(\mathrm{PDI}=0.272)$ and zeta potentials were $-0.2 \mathrm{mv}$, which changed obviously after drug releasing (Fig. 1b). While compared with other groups in Fig. 1c, it can be vividly observed that the second peak (CA4) appeared after the ester bond was broken and CA4 released from nanoparticles in $\mathrm{pH} 5.5$, which all proved that PEG-b-PAsp-g-CA4 had a better release in the TME directly. The final PEG-b-PAsp-gCA4 conjugates had a CA4 drug loading content (DLC\%) of $29.8 \mathrm{wt} \%$ as confirmed by UV-Vis spectrometry. The quantity of CA4 released in $\mathrm{pH} 7.4, \mathrm{pH} 5.0$ PBS buffer or distilled water was quantified by HPLC using a UV-Vis detector, as shown in Fig. 1e. CA4 releasing from CA4NPs was $47.2 \pm 2.3 \%$ at $48 \mathrm{~h}$ in $\mathrm{pH} 5.5 \mathrm{PBS}$ buffer, greater than in pH 7.4 PBS buffer $(27.3 \pm 1.3 \%)$, and in distilled water $(16.2 \pm 2.1 \%)$. The CA4 releasing at lower $\mathrm{pH}$ was quick in $4 \mathrm{~h}$ and then became slow in next $44 \mathrm{~h}$ in $\mathrm{pH} 5.5$, which revealed that the ester bond of was acid-sensitive to the TME and PEG-b-PAsp-g-CA4 can reach the concentration of the drug quickly and last long next.

\section{In vitro cytotoxicity and uptake of the CA4-NPs experimental section}

Cytotoxicity of the different drugs was respectively evaluated by MTT assay against Hepa1-6, HepG2, LO2 cells, and primary hepatocytes were used in this study. As shown in Fig. 2a, 0.0025-25 $\mu$ M of CA4, CA4P, CA4 -NPs exhibited cytotoxicity to Hepa1-6 and HepG2 cells. Not like small molecules, the release of drug from nanoparticles was sustained and controlled. As a result, the cytotoxicity of CA4 -NPs was lower than CA4 in limited time because the drugs linked with nanoparticles have not been completely released to exert their effects on cells. Meanwhile, the prepared CA4-NPs showed no cytotoxicity to LO2 cells and primary hepatocytes, which meant that PEG-b-PAsp can be used as a safe drug carrier.

To visualize the intracellular behavior of CA4 -NPs, we labeled the nanomaterials by fluorescein isothiocyanate (FITC) and investigated their uptake by Hepa 1-6 cells using confocal laser scanning microscopy (CLSM) and fluorescence-activated cell sorting (FACS). The 
a

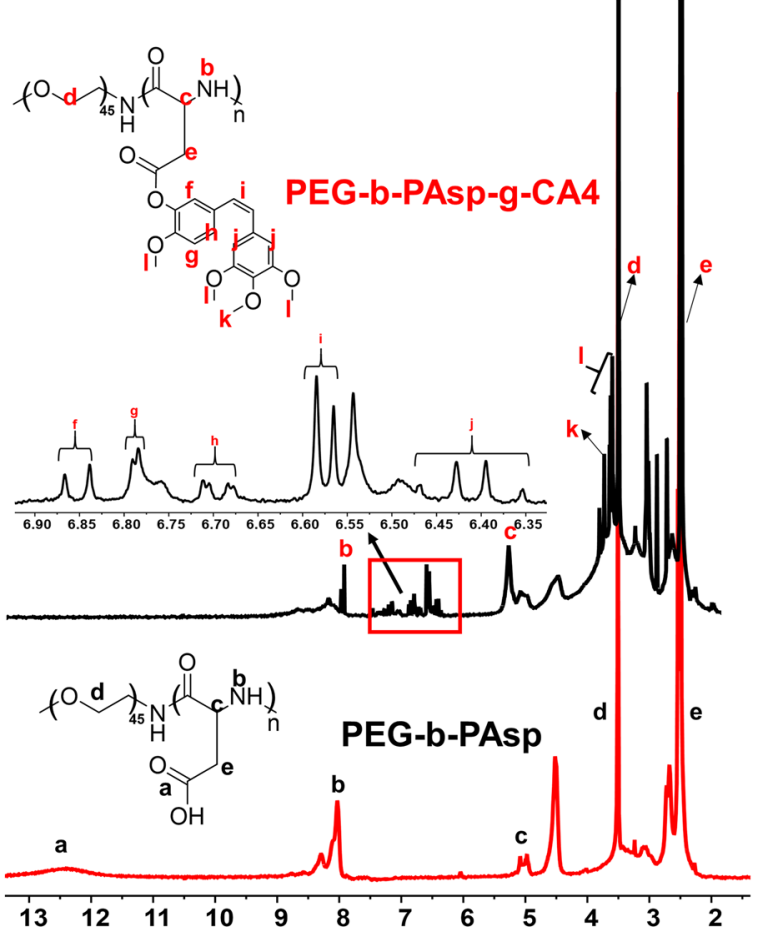

c
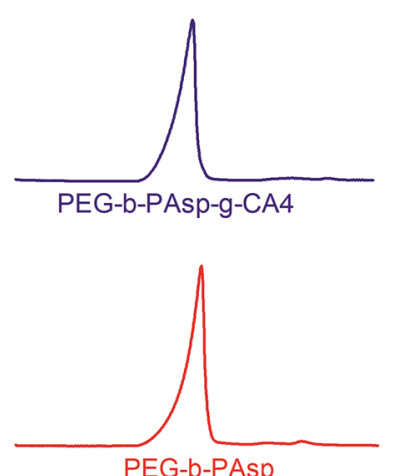

PEG-b-PAsp

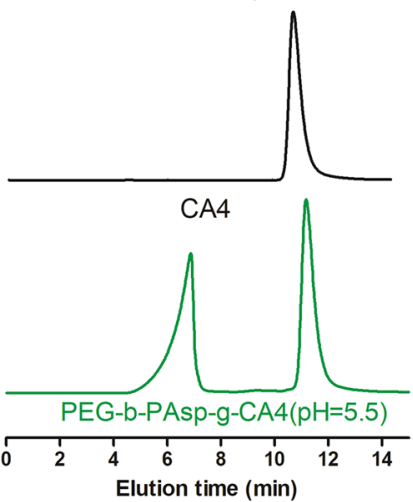

b

PEG-b-PAsp

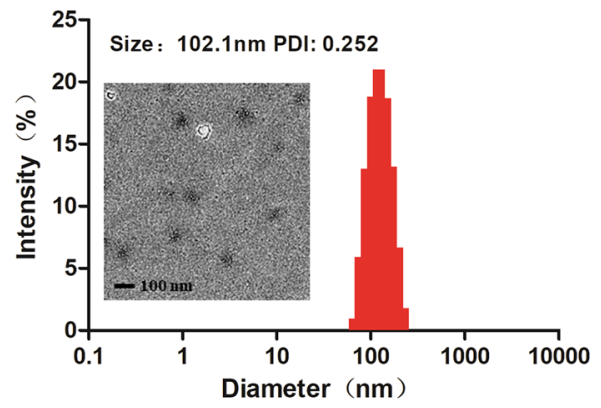

PEG-b-PAsp-g-CA4 ( $\mathrm{pH}=5.5)$

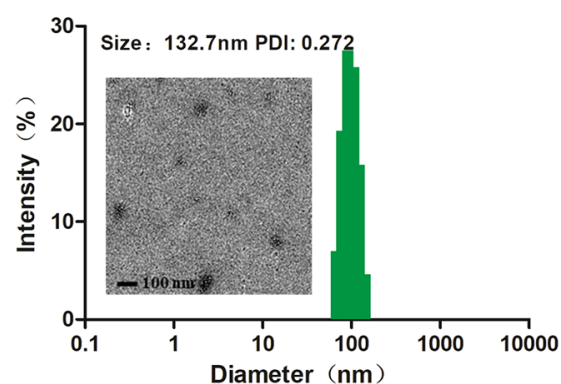

PEG-b-PAsp-g-CA4
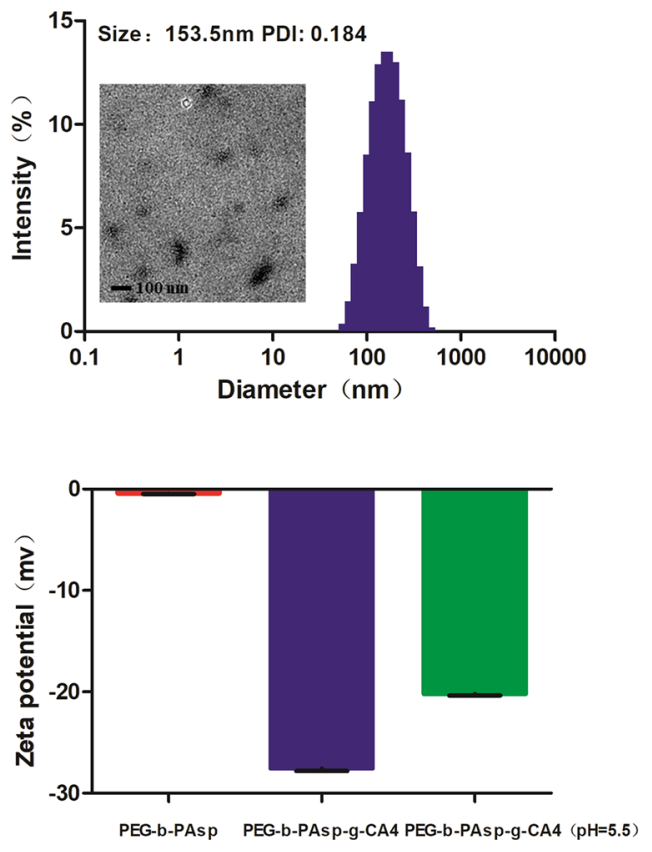

Fig. 1 a ${ }^{1} H$ NMR spectra of PEG-b-PAsp and PEG-b-PAsp-g-CA4; b Representative TEM images, size distribution, and zeta potential of the PEG-b-PAsp and PEG-b-PAsp-g-CA4 (also including in pH =5.5); c GPC curves of PEG-b-PAsp-g-CA4, PEG-b-PAsp, CA4, and PEG-b-PAsp-g-CA4 (in $\mathrm{pH}=5.5$ ); $\mathbf{d}$ Critical micellar concentration measurement of PEG-b-PAsp and PEG-b-PAsp-g-CA4 in water; $\mathbf{e}$ UV-vis absorption spectra of CA4 and drug release of PEG-b-PAsp-g-CA4 in pH 5.5/ 7.4 PBS buffer, distilled water 
d

PEG-b-PAsp

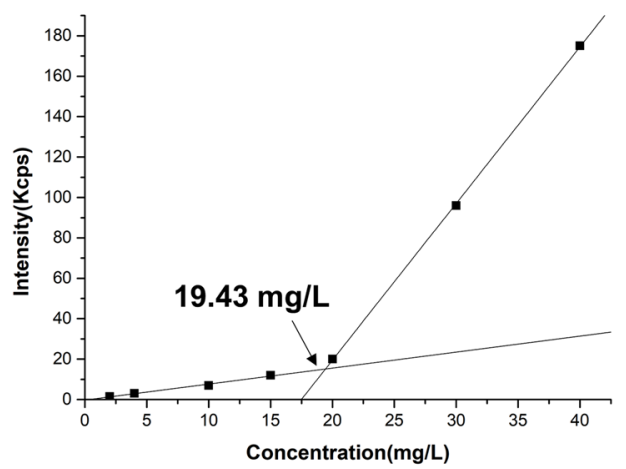

e

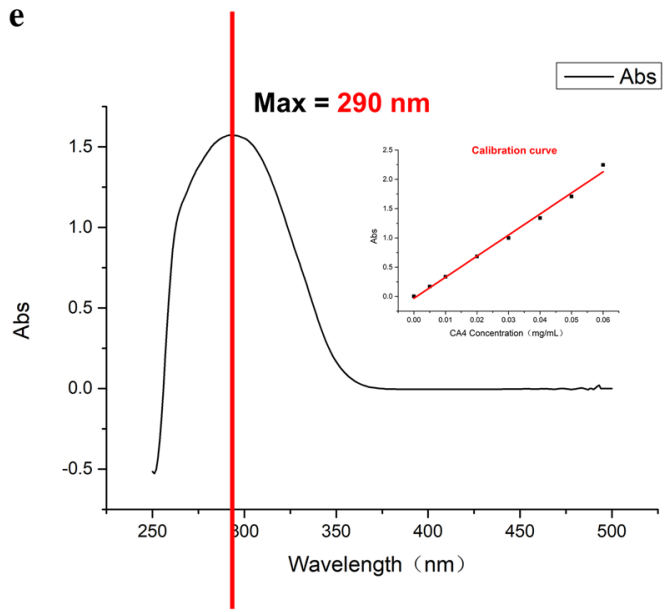

PEG-b-PAsp-g-CA4
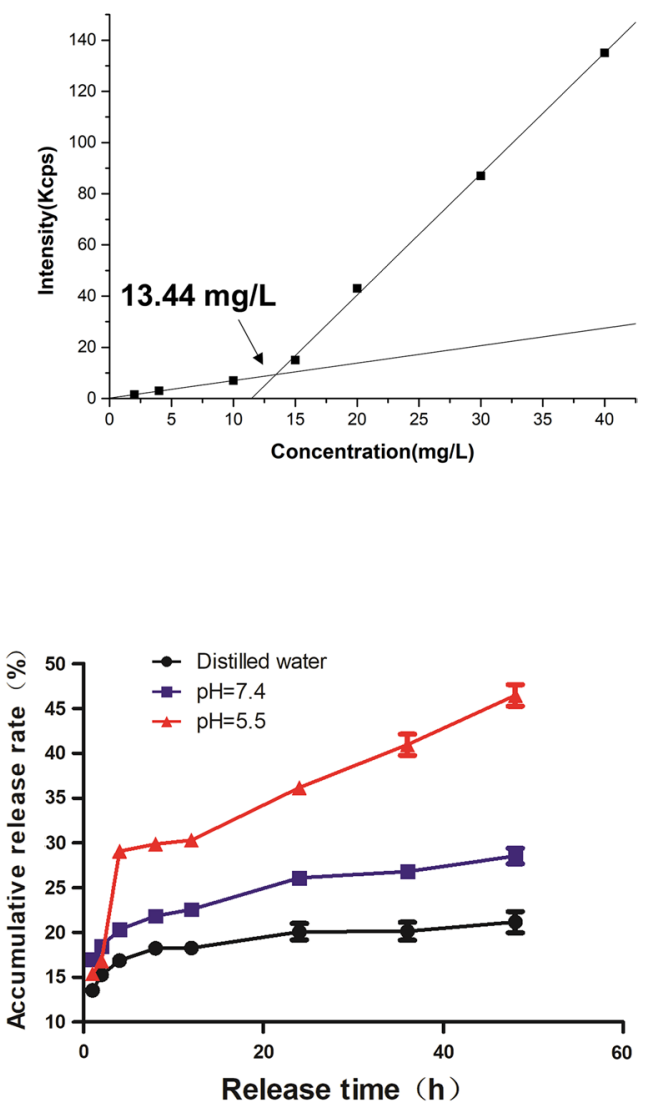

Fig. 1 continued

concentration of FITC and PEG-b-PAsp-g-CA4-FITC (on the FITC basis) varied from 10 to $40 \mu \mathrm{g} \mathrm{mL}^{-1}$ and the incubated time with Hepa1- 6 cells was 2, 4, 6 h. The highest cell positive rate was over $90 \%$ detected by flow cytometry $4 \mathrm{~h}$ later and at a concentration of $40 \mu \mathrm{g} \mathrm{mL}$ of CA4 -NPs. Nonetheless, a lower cell positive rate was obtained after being incubated after $6 \mathrm{~h}$ than it after $4 \mathrm{~h}$, which may result from the enhanced cytotoxicity of FITC after being incubated longer time (Fig. 2b). And dependent on this condition, cellular localization experiments were performed using CLSM. Hepa1-6 cells treated with PEG-b-PAsp-g-CA4-FITC $\left(40 \mu \mathrm{g} \mathrm{mL}^{-1}\right)$ presented significantly higher fluorescent signal after $4 \mathrm{~h}$ in Fig. 2c, indicating a more cellular uptake than that of free FITC in the nuclei.

\section{Pharmacokinetics and biodistribution of CA4-NPs}

To compare the circulation time of CA4 -NPs, and CA4P in vivo, SD rats were administered with CA4-NPs or CA4P at a dose of $50.0 \mathrm{mg} \mathrm{kg}^{-1}$ based on CA4. As shown in Fig. 3a, the plasma concentration-time profiles fitted in Non-compartment models well, and the main pharmacokinetic parameters of PEG-b-PAsp-g-CA4 were as follows: $\mathrm{C}_{\max } 50.96 \pm 5.41 \mu \mathrm{g} \mathrm{mL}-1, \mathrm{~T}_{1 / 2} 2.18 \pm 0.22 \mathrm{~h}$, MRT $2.26 \pm 0.17 \mathrm{~h}, \mathrm{AUC}_{0-\tau} 120.66 \pm 10.37 \mathrm{mg} \mathrm{h}^{-1} \mathrm{~L}^{-1}$, $\mathrm{AUC}_{0-\infty} 123.21 \pm 10.85 \mathrm{mg} \mathrm{h}^{-1} \mathrm{~L}^{-1}$. In contrast, for free CA4P, $C_{\max } 49.18 \pm 1.02 \mu \mathrm{g} \mathrm{mL}{ }^{-1}, \mathrm{~T}_{1 / 2} 0.24 \pm 0.02 \mathrm{~h}$, MRT $0.28 \pm 0.02 \mathrm{~h}, \mathrm{AUC}_{0-\mathrm{\tau}} 15.32 \pm 1.49 \mathrm{mg} \mathrm{h}^{-1} \mathrm{~L}^{-1}$, $\mathrm{AUC}_{0-\infty} 15.51 \pm 1.56 \mathrm{mg} \mathrm{h}^{-1} \mathrm{~L}^{-1}$. The pharmacokinetic data indicated that PEG-b-PAsp-g-CA4 has a longer blood circulation time than CA4P, achieving more tumor accumulation and retention to destroy tumor blood vessels for a longer period, which can provide higher antitumor efficacy by administering lower doses at longer intervals. Pharmacokinetic data was analyzed using WinNonLin software version 7.0.

To verify the behaviors in vivo, free Cyanine 5.5 NHS ester (Cy5.5, SE) and PEG-b-PAsp-g-CA4-Cy5.5 were monitored by in vivo imaging method. As shown in Fig. 3b, free Cy5.5 had no significant tumor accumulation 


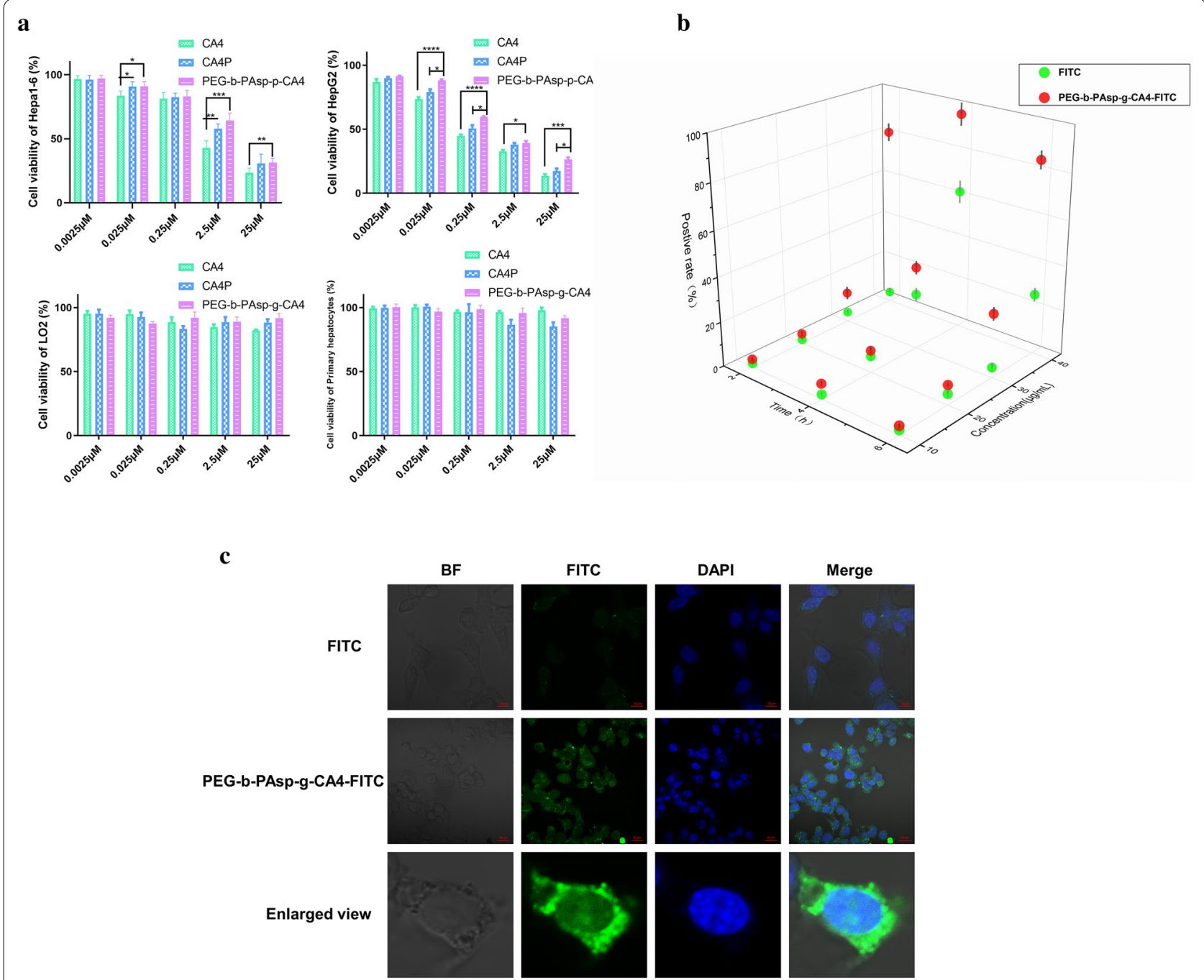

Fig. 2 The cytotoxicity and uptake of the CA4-NPs. a Cytotoxicity of CA4, CA4P, PEG-b-PAsp-g-CA4 to Hepa1-6, HepG2, primary hepatocytes, LO2; b Fluorescence intensity of positive Hepa1-6 cells mixed with FITC and PEG-b-PAsp-g-CA4-FITC under different condition (Concentration of FITC and PEG-b-PAsp-g-CA4-FITC (on the FITC basis) varied from 10 to $40 \mu \mathrm{g} \mathrm{mL}^{-1}$ and incubated time was 2, 4, 6 h.). Cellular uptake was analyzed by fluorescence-activated cell sorting (FACS); c Confocal images of Hepa1-6 cells after a $4 \mathrm{~h}$ incubation with $40 \mu \mathrm{g} \mathrm{FITC} \mathrm{mL}^{-1}$ and PEG-b-PAsp-g-CA4-FITC (on the FITC basis). Scale bar: $20 \mu \mathrm{m}$. Significance between each pair of groups was calculated using Student's t-test. ${ }^{*} p<0.05,{ }^{* *} p<0.01,{ }^{* * *} p<0.001,{ }^{* * *} p<0.0001$. The values are presented as the mean $\pm S D, n=6$

at each predetermined time. Notably, the fluorescence of Cy5.5 delivered by PEG-b-PAsp-g-CA4 peaked at around $6 \mathrm{~h}$. The fluorescence intensity of PEG-b-PAsp-g-CA4Cy5.5 remained about 4 times higher in tumors ex vivo that of free Cy5.5 at $24 \mathrm{~h}$ after administration ( $<<0.001$, Fig. 3c, d). This phenomenon can be explained by the EPR effect and acid-sensitive $(\mathrm{pH}=5.5)$ effect of CA4 NPs to TME, and the excellent selectivity of CA4 for tumor central blood vessels, which is conducive for CA4NPs to exert a better therapeutic effect compared with the CA4 treatment group (Additional file 1: Figure S1). At the stage of drug accumulation in vivo, free molecule showed a little bit faster elimination for overall observation in the major organs (heart, liver, spleen, lungs, and kidneys) when compared to the NPs group in Fig. $3 \mathrm{~d}$ and Additional file 1: Figure S2. In summary, the CA4-NPs was able to efficiently accumulate and retain in tumors after intravenous injection, which could be beneficial for anticancer applications in vivo.

\section{Mechanism of combination aPD-L1 with CA4-NPs}

In order to investigate the vascular state after $48 \mathrm{~h}$ post-injection of CA4-NPs, we used immunofluorescence technology to stain CD31 of tumor tissues, which 
a

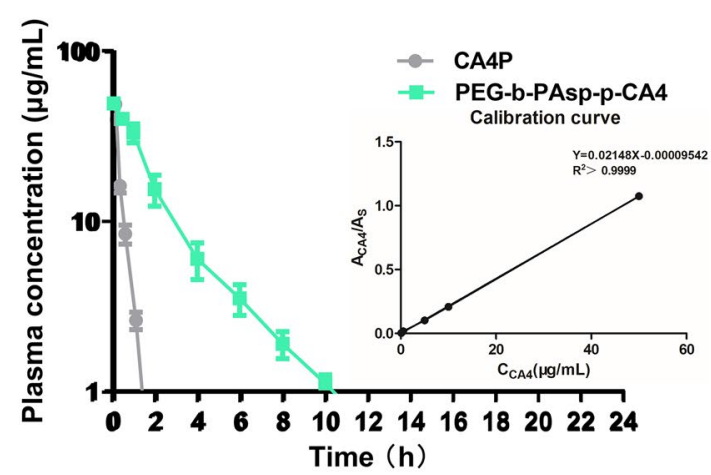

c

PEG-b-PAsp-p-CA4-Cy5.5

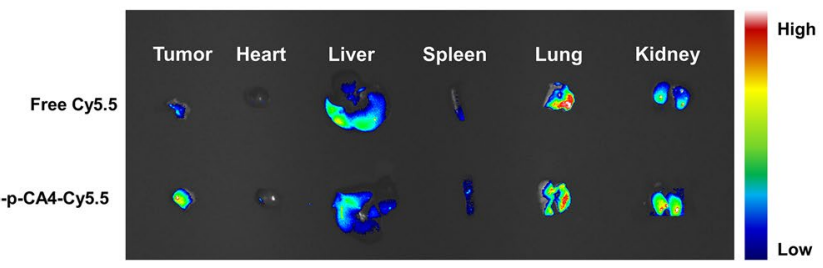

b

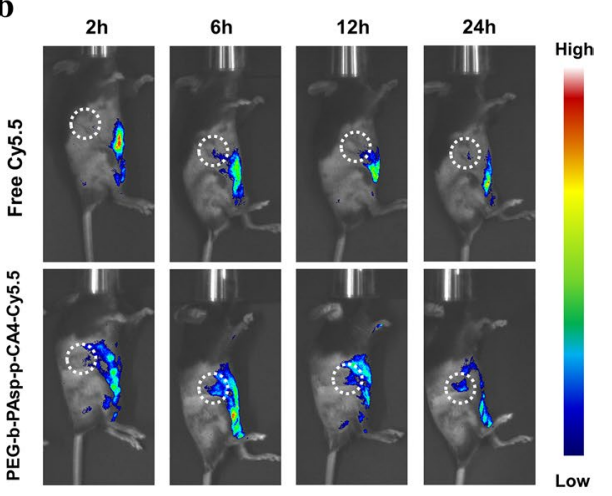

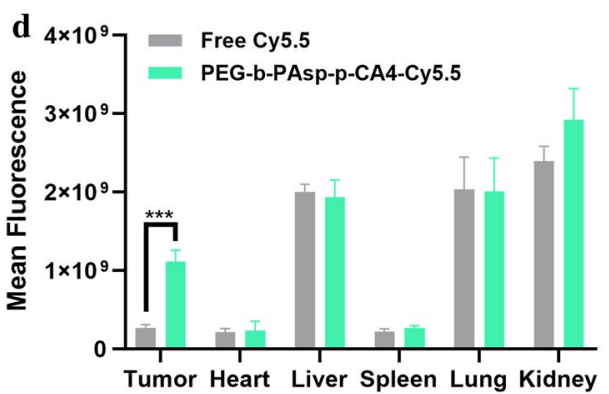

Fig. 3 Pharmacokinetics and biodistribution of CA4-NPs. a Pharmacokinetics of CA4P and PEG-b-PAsp-g-CA4; $\boldsymbol{b}$ In vivo fluorescence images of Hepa 1-6 tumor-bearing mice taken at different time points intravenously administrated with Cy5.5 and PEG-b-PAsp-g-CA4-Cy5.5; c Ex vivo fluorescence images of the major organs (heart, liver, spleen, lungs, and kidneys) and tumors at $24 \mathrm{~h}$ intravenous administration; $\mathbf{d}$ Quantitative analysis of ex vivo Cy5.5 fluorescence intensity of the organs and tumor. Significance between each pair of groups was calculated using Student's t-test. ${ }^{*} p<0.05,{ }^{* *} p<0.01,{ }^{* * *} p<0.001,{ }^{* * *} p<0.0001$. The values are presented as the mean $\pm S D, n=3$

indicated that the vascular density in the CA4-NPs (40) group was lower than that in the CA4-NPs (20) group, and the vascular density in the CA4-NPs (20) group was lower than that in the PBS group (Fig. 4a). These results showed that CA4-NPs performed good triple target effects-EPR effect, acid-sensitive $(\mathrm{pH}=5.5)$ effect to TME, and high selectivity for tumor blood vessel, and strong dose-dependent vascular disruption in tumor tissues. Considering that CA4-NPs treatment could induce a severe hypoxic microenvironment in solid tumors because of vascular disruption, we then compared the level of HIF-1 $\alpha$ in Hepa1-6 tumor tissues under immunofluorescence staining. As a result, CA4-NPs treatment increased the expression of HIF- $1 \alpha$ in tumor tissues significantly. The expression of HIF- $1 \alpha$ in the CA4-NPs (20) group was higher than that in the PBS group, and the level of HIF-1 $\alpha$ in the CA4-NPs (40) group was the highest among the three groups after $48 \mathrm{~h}$ intravenous injection (Fig. 4b). These results revealed that CA4-NPs treatment could block the vessels and induce severe hypoxic conditions that HIF-1 $\alpha$ expressed higher in tumor tissues. Thus, we continued to compare the level of PD-L1 in Hepa1-6 tumor tissues dependent on the results of HIF- $1 \alpha$, and a similar conclusion was obtained that CA4-NPs treatment also increased the expression of PD-L1 in tumor tissues significantly. Nonetheless, the level of PD-L1 in the combined treatment (CA4NPs with aPD-L1) significantly descended compared to the single administration group (CA4-NPs) after $48 \mathrm{~h}$ intravenous injection, and single administration group (aPD-L1) showed the most obviously inhibitory effect on PD-L1 expression level among the six groups (Fig. 4c), which revealed that CA4-NPs together with aPD-L1 might produce a better treatment effect compared to the single administration.

\section{In vivo anti-tumor efficacy}

It is not ideal for tumor treatment when the overexpression of PD-L1 in tumor tissues induced by hypoxic microenvironment after the use of CA4-NPs, which is quite possible to result in reoccurrence of tumor cells. To address this serious problem, we combined aPD-L1 with CA4-NPs simultaneously dependent on the results of the pharmacokinetics and biodistribution of CA4-NPs and exploration of mechanism of co-administration with aPD-L1 before. A subcutaneous Hepa1-6 hepatocellular 
carcinoma mouse model was established on female C57BL/6 mice as described above.

When the tumor volume reached about $150 \mathrm{~mm}^{3}$, the mice were randomly divided into 6 groups: PBS, CA4-NPs (20 mg kg-1), CA4-NPs (40 mg kg-1), aPDL1, CA4-NPs $\left(20 \mathrm{mg} \quad \mathrm{kg}^{-1}\right)+\mathrm{aPD}-\mathrm{L} 1, \quad$ CA4-NPs
(40 mg kg-1)+aPD-L1 (20 mg kg-1). CA4-NPs (20, $40 \mathrm{mg} \mathrm{kg}{ }^{-1}$ ) and PBS were separately administrated to mice via intravenous injection at day 1, 8 and aPD-L1 was administrated to mice via intratumoral injection at day $1,4,7,10,13$, and the tumor volumes were continuously monitored for 15 days (Fig. 5a). The dosage of aPD-L1 a
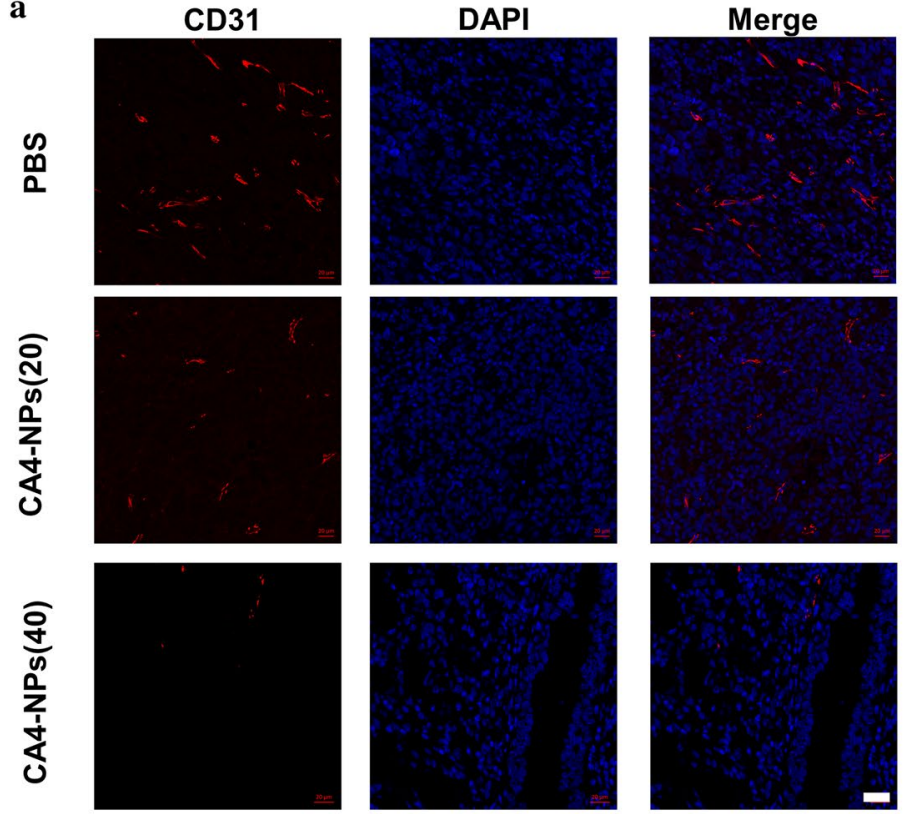

b
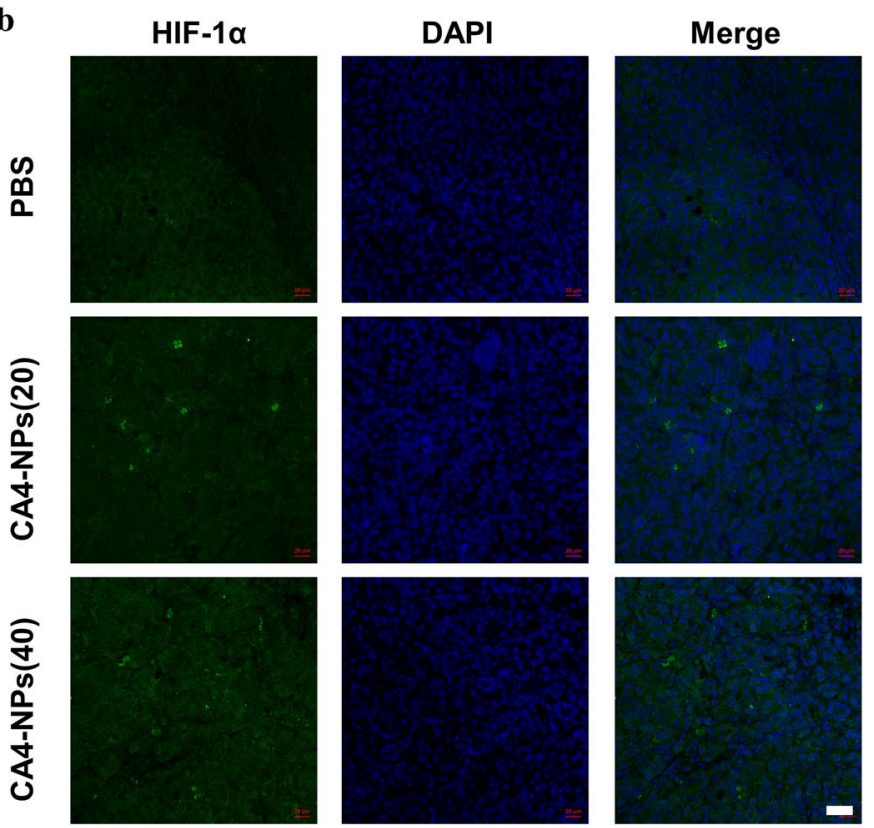

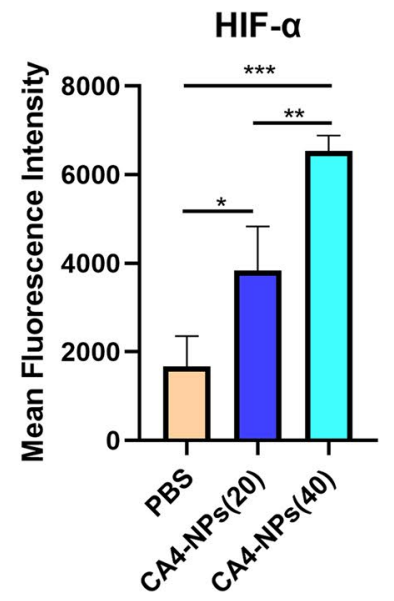

Fig. 4 Mechanism of combination aPD-L1 with CA4-NPs. Immunofluorescence staining for a CD31, b HIF-1a, c PD-L1 expression of tumor tissues $48 \mathrm{~h}$ post-treatment and quantitative immunofluorescence analysis. CA4-NPs (20) represented that the dose of CA4-NPs was $20 \mathrm{mg} \mathrm{kg}^{-1}$ and CA4-NPs (40) represented that the dose of CA4-NPs was $40 \mathrm{mg} \mathrm{kg}^{-1}$. Scale bar: $20 \mu \mathrm{m}$. Significance between each pair of groups was calculated using Student's t-test. ${ }^{*} p<0.05,{ }^{* *} p<0.01,{ }^{* * *} p<0.001,{ }^{* * *} p<0.0001$. The values are presented as the mean $\pm S D, n=3$ 

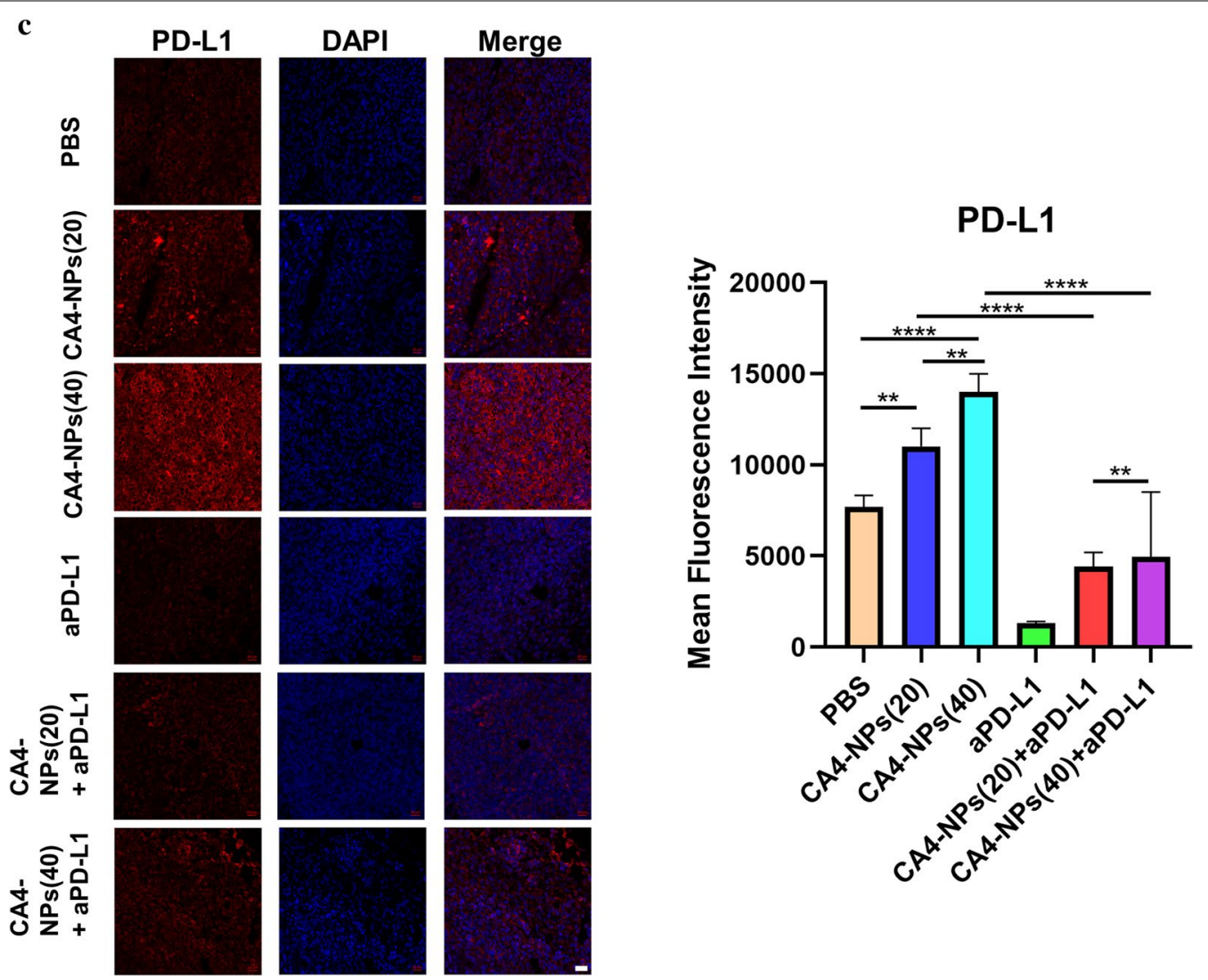

Fig. 4 continued
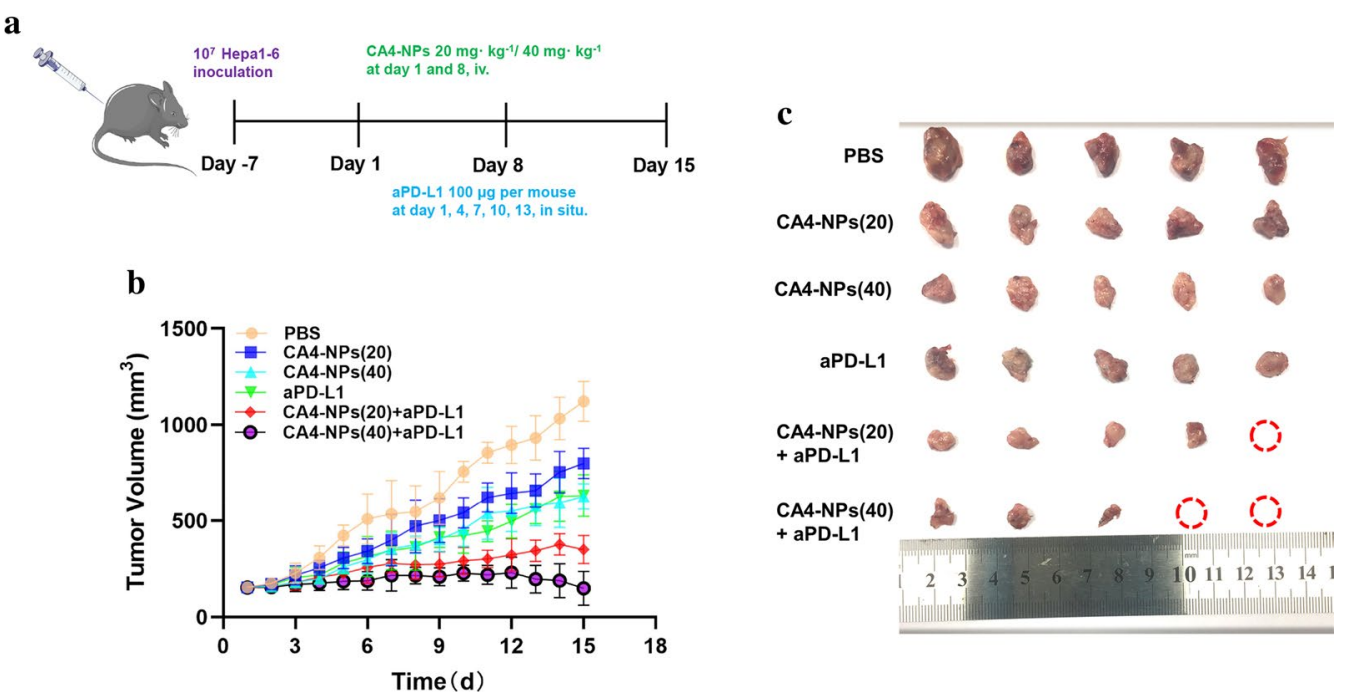

Fig. 5 Co-administration of anti-tumor efficacy In vivo. a Treatment scheme of subcutaneous Hepa1-6 tumors with CA4-NPs and aPD-L1; $\mathbf{b}$ Tumor volume growth curves following different treatments; c Representative images of the excised tumors after different treatments; shown are PBS-treated mice and mice receiving after injection with CA4-NPs $\left(20 \mathrm{mg} \mathrm{kg}^{-1}\right)$, CA4-NPs $\left(40 \mathrm{mg} \mathrm{kg}^{-1}\right)$, aPD-L1, CA4-NPs $\left(20 \mathrm{mg} \mathrm{kg}{ }^{-1}\right)+$ aPD-L1, CA4-NPs (40 $\left.\mathrm{mg} \mathrm{kg}^{-1}\right)+$ aPD-L1; d Tumor weights from the different groups on day 15; e The survival percentages of the tumor-bearing C57BL/6 mice $\left(n=7\right.$ biologically independent samples). Significance between each pair of groups was calculated using Student's t-test. ${ }^{*} p<0.05,{ }^{* *} p<0.01$, ${ }^{* * *} p<0.001,{ }^{* * *} p<0.0001$. The values are presented as the mean $\pm S D, n=10$ 


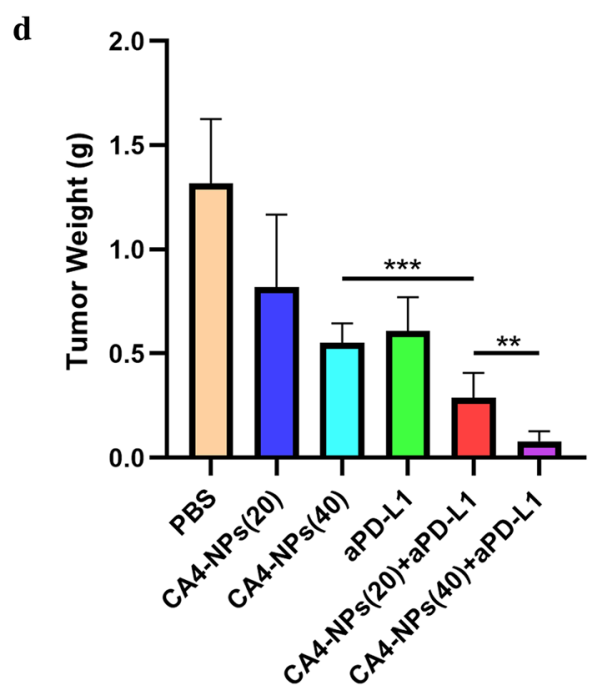

e

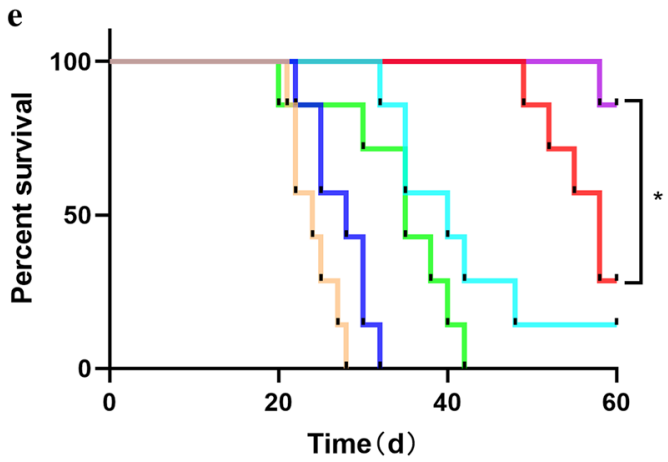

Median Survival (days)

\section{4}

28

40

35

58

not reached

Fig. 5 continued

was $50 \mu \mathrm{L}$ for each mouse. Tumor growth curves were shown in Fig. 5b where fast-growing tumor volumes were observed in PBS and CA4-NPs (20) treated mice. In contrast, a modest inhibition of tumor growth was achieved in mice receiving CA4-NPs (40) and aPD-L1, and also as shown in Fig. 5 c, d, a $58.1 \%$ or $53.7 \%$ reduction of the relative tumor weight at 15 th day was observed respectively. Notably, the most effective anti-tumor effect was realized in CA4-NPs $(20,40)$ with aPD-L1 treated group that tumor weight at the last day was reduced by $78.2 \%$ and $89.7 \%$, which may be benefited from the combined blocking effect of aPD-L1 in TME.

As depicted in Fig. 5e, the median survival time of mice receiving CA4-NPs (20) and CA4-NPs (40) were 28 and 40 days, respectively. The combination treatment significantly prolonged survival of Hepa1-6 tumor-bearing mice compared with the single drug treatment groups. The median survival time of mice in the combination groups was more than 58 days. The above observations unambiguously evidenced the remarkable anticancer efficacy of the combinenation strategy.
Additionally, to investigate the anti-tumor therapeutic mechanism of the combined treatment, histological staining analyses were applied to the isolated tumors, including Ki67, TUNEL and hematoxylin and eosin (H\&E). Less Ki67-positive cells (proliferative cells) and more TUNEL-positive cells (apoptotic cells) could be found in the co-administration treated tumors (Fig. 6a). On the contrary, more proliferative tumor cells and fewer apoptotic tumor cells were observed in the case of PBS and single administration treated mice. We next analyzed CD31 expression in tumors for immunohistochemistry (IHC) to investigate tumor microvascular changes after different treated groups. The CD31 staining images of mice in CA4-NPs group were taken in the peripheral area of the tumor (Fig. 6b). CA4-NPs significantly disrupted the established tumor vascular and suppressed tumor growth (inner area), while continuous administration groups of aPD-L1 showed obviously inhibitory revascularization and regrowth of the treated tumor (peripheral area), which may be contributed to the treatment of tumor reoccurrence. Also, the number of $\mathrm{CD}^{+} \mathrm{T}$ cells 
$\mathbf{a}$
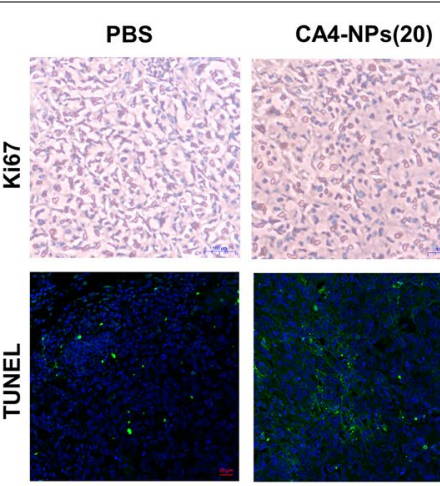

CA4-NPs(20)

CA4-NPs(40)

aPD-L1

CA4-NPs(20) + aPD-L1 CA4-NPs(40) + aPD-L1

b

PBS
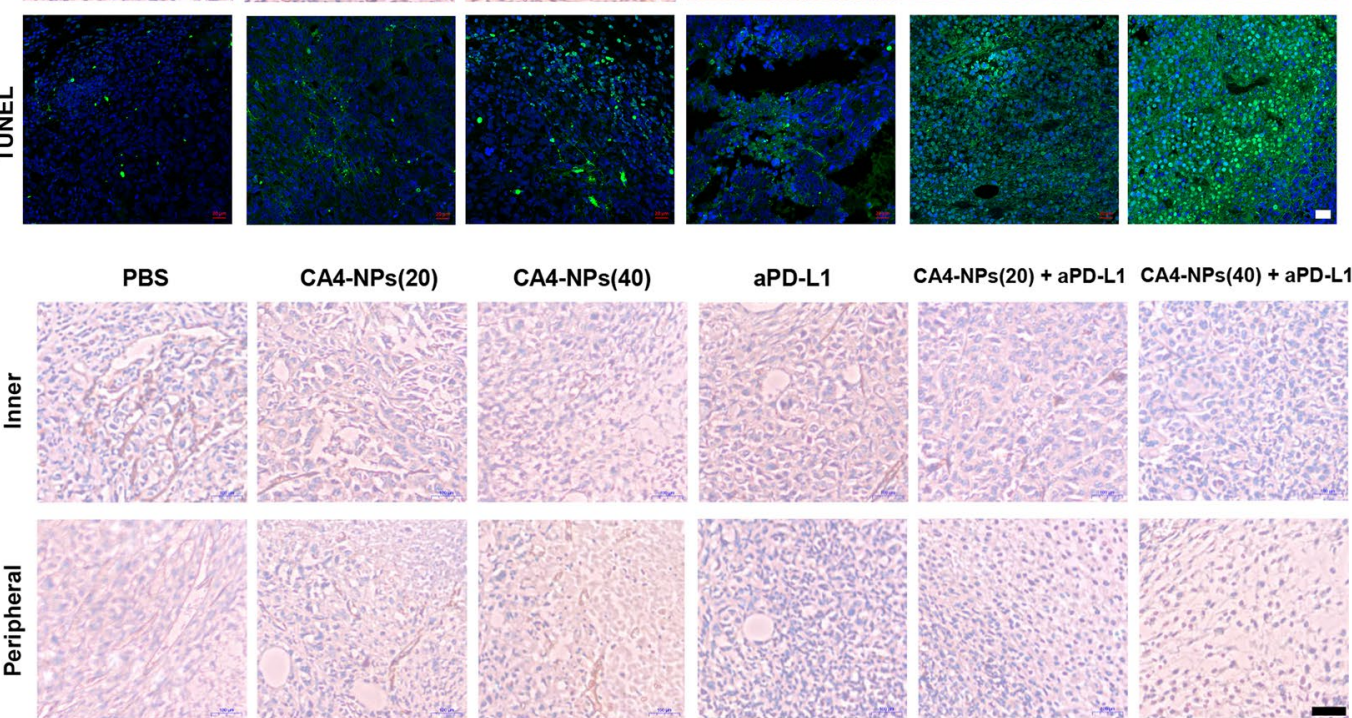

CA4-NPs $(40)$

aPD-L1

CA4-NPs(20) + aPD-L1 CA4-NPs(40) + aPD-L1
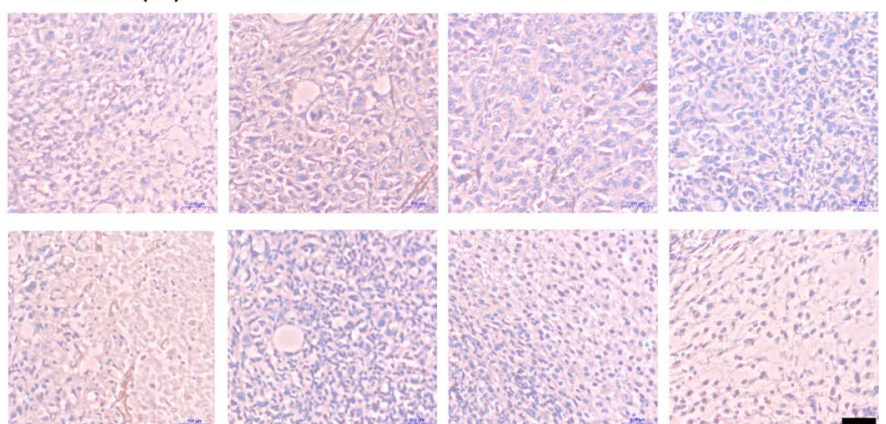

c
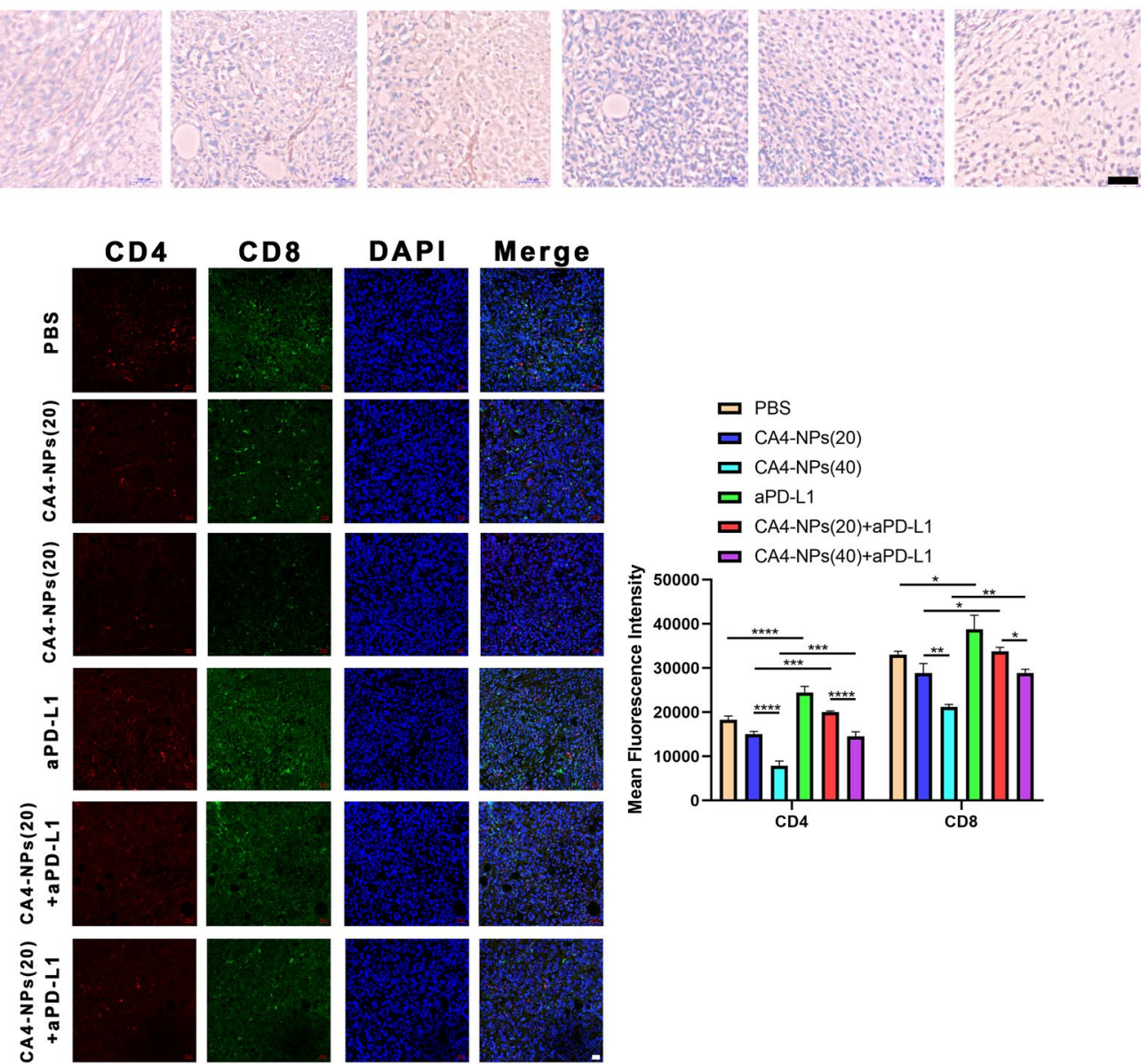

Fig. 6 a Ki67 and TUNEL staining of tumor tissues after different treatments; $\mathbf{b}$ Immunohistochemical reactivity for blood vessel endothelial cell marker CD31 in subcutaneous Hepa1-6 bearing mice; c Double-color immunofluorescence staining for CD4 (Red) and CD8(Green) expression of tumor tissues, and quantitative immunofluorescence analysis; $\mathbf{d}$ The secretions of cytokines in serum and tumor site were measured by ELISA (levels of IFN- $\gamma$ and IL-4); e Immunofluorescence staining for LAG-3 and TIM-3 expression of tumor tissues, and quantitative immunofluorescence analysis. Black scale bar $=100 \mu \mathrm{m}$ and white scale bar $=20 \mu \mathrm{m}$. Significance between each pair of groups was calculated using Student's t-test. ${ }^{*} p<0.05$, ${ }^{* *} p<0.01,{ }^{* * *} p<0.001,{ }^{* * * *} p<0.0001$. The values are presented as the mean $\pm S D, n=3$ 


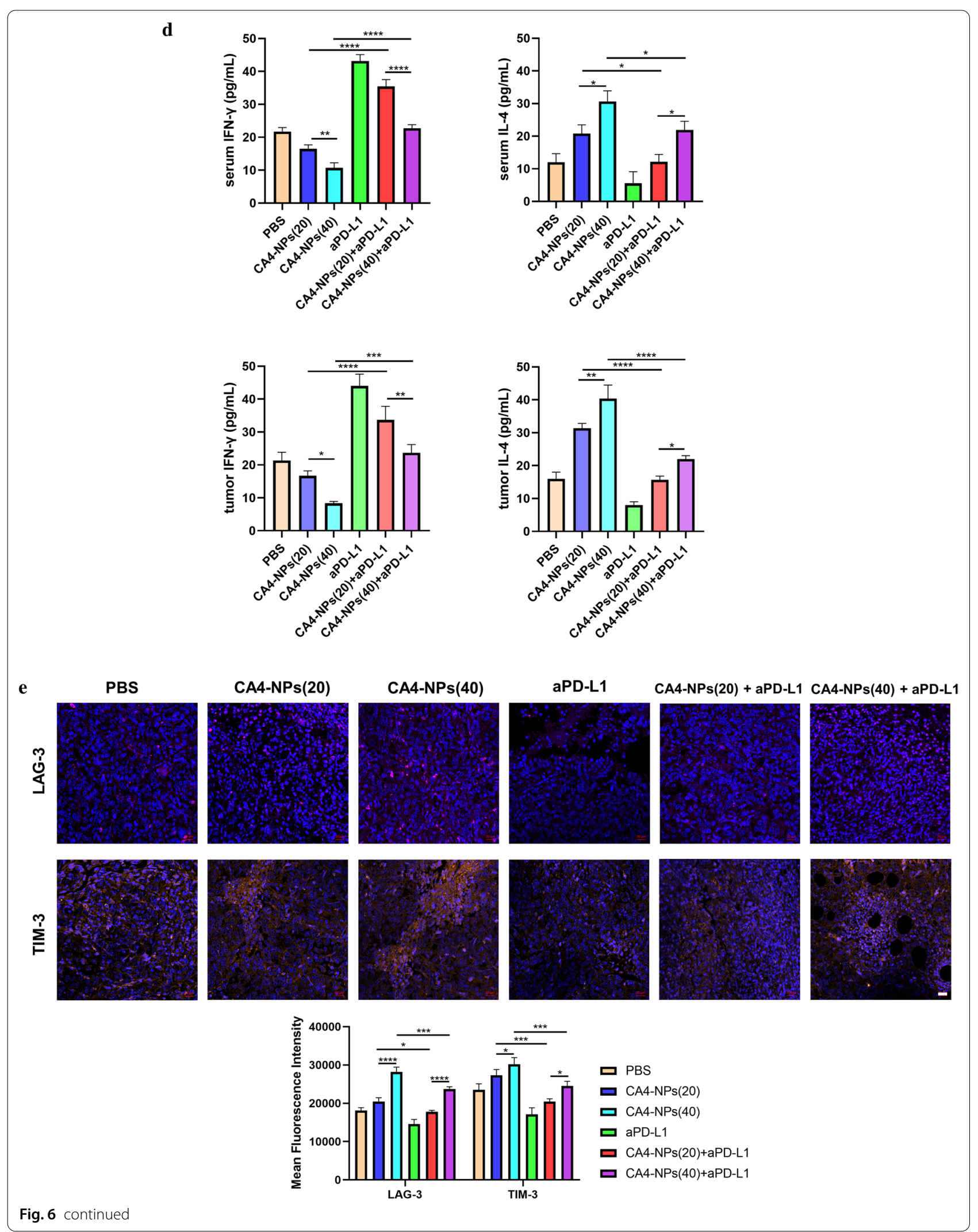


and $\mathrm{CD}^{+} \mathrm{T}$ cells were significantly increased in combination groups when compared to single groups (Fig. 6c). Previous research suggested that IFN- $\gamma$ and IL-4 are representative of Th1 and Th2 cytokines [35]. The production of signature Th1 cytokine IFN- $\gamma$ increased more both in serum and tumor site by combinated strategy, whereas for the Th2 cytokine IL-4, it decreased significantly in combination groups in Fig. 6d. It is also widely reported that the expression of Tim-3 and Lag-3 on different tumor cells can be served as potential biomarkers to predict the response to anti-PD-1/PD-L1 therapy [36-38]. So we finally investigated the LAG-3 and TIM-3 expression in tumor tissues by immunofluorescence staining and it can obserbed that tumors treated by CA4NPs expressed less LAG-3/TIM-3 after combinatd with aPD-L1 in Fig. 6e. All these mechanism results confirmed the successful anti-tumor therapy by co-administration. Furthermore, in Fig. 7, compact tumor tissue was found for PBS and CA4 treated mice, while a remarkable reduction of tumor cells and more vacancies was observed after co-administration. Meanwhile, no significant loss of body weight was observed in all groups, indicating the safety and biocompatibility of the nanoparticles used here, which was further confirmed by $H \& E$ staining of major organs.

\section{Conclusion}

In this study, a combination of poly(L-Aspartic acid)poly(ethylene glycol)/combretastatin A4 (CA4-NPs) nanoparticles with aPD-L1 was developed for systemic treatment of HCC. The use of CA4-NPs was aimed to cause extensive tumor necrosis mainly due to the triple target effects-EPR effect, acid-sensitive $(\mathrm{pH}=5.5)$ effect to TME and good selectivity of CA4 for tumor blood vessel. Also, the circulation time of CA4-NPs in vivo was about 10 times that of CA4P. However, CA4-NPs also induced overexpression of PD-L1 that was confirmed by immunofluorescence staining in the treated tumors. Therefore, the aPD-L1 was applied to efficiently up-regulate the growth and proliferation of $\mathrm{T}$ cells and activate their attack and killing functions. The combination of PEG-b-PAsp-g-CA4 at $40 \mathrm{mg} \mathrm{kg}^{-1}$ with aPD-L1 $(50 \mu \mathrm{L})$ showed a nearly $90 \%$ tumor suppression rate in a Hepa1-6 subcutaneous tumor model with low systemic toxicity. These findings indicated that the two-pronged attack of aPD-L1 with a strategy of VDAs, CA4-NPs, was really a potential therapeutic approach for HCC treatment.

\section{Methods \\ Materials}

All reagents and solvents were commercially available and used without additional treatment. L-Aspartic acid benzyl ester was purchased from Shanghai Bide Pharmatech Co., LTD. Triphosgene was purchased from Shanghai Aladdin Biotechnology Co., LTD. Combretastatin A4 (CA4) and combretastatin-A4 phosphate (CA4P) were purchased from Nanjing Chunqiu Biotechnology Co., LTD. aPD-L1 (BE0101) used in vivo purchased from Bio X Cell. Cyanine5.5 NHS ester (Cy5.5, SE) was purchased from Beijing Fanbo Biochemicals Co., LTD. Dulbecco's modified Eagle's medium (DMEM), fetal bovine serum (FBS), 3-(4,5-dimethylthiazol-2-yl)-2,5-diphenyltetrazolium bromide (MTT), trypsin-EDTA, penicillin-streptomycin, dimethyl sulfoxide (DMSO), and 4,6-diamidino-2-phenylindole (DAPI) were obtained from Gibco. 96 well plates, 6-well plates, and $10 \mathrm{~mL}$ graduated sterile centrifuge tubes were purchased from Jiangsu KeyGen Biotechnology Co., LTD. Other reagents and chemicals were at least analytical reagent grade.

\section{Preparation of PEG-b-PAsp-g-CA4}

L-Aspartic acid benzyl ester and triphosgene $\left(\mathrm{C}_{3} \mathrm{Cl}_{6} \mathrm{O}_{3}\right)$ were added into anhydrous THF respectively, then were mixed, stirred, and heated for $2 \mathrm{~h}$ at $55^{\circ} \mathrm{C} . \mathrm{N}$-carboxy-a-amino acid anhydrides of b-Benzyl-L-aspartate (BLA-NCA) was obtained after being added n-hexane for crystallization and suction. The diblock copolymer (PEG-b-PAsp) was firstly synthesized via the amineinitiated ring-opening polymerization (ROP) of BLANCA. Briefly, BLA-NCA was dissolved completely in DMF/DCM followed by the addition of initiator which had been dissolved in DCM. Then, the reaction mixture was stirred for 5 days at $35^{\circ} \mathrm{C}$ under a dry nitrogen atmosphere and the crude products were precipitated in tenfold excess of cold diethyl ether and isolated by centrifugation. After washed twice with diethyl ether, the products were dried in a vacuum. Block copolymers of PEG-b-PAsp were obtained by deprotection of PEGPBLA in $\mathrm{HCCl}_{2} \mathrm{COOH} / \mathrm{HBr} / \mathrm{HAc}$ solution. After stirred for $3 \mathrm{~h}$ under an ice bath, the solution was precipitated in a large amount of cold diethyl ether and isolated by centrifugation. The solid was dissolved in DMSO. The solution was dialyzed against distilled water (MWCO 3500) and then solid PEG-b-PAsp was obtained by lyophilization. CA4 was grafted to PEG-b-PAsp by the Yamaguchi reaction. Briefly, PEG-b-PAsp was dissolved in anhydrous $\mathrm{N}, \mathrm{N}$-dimethylformamide (DMF) in a glass reactor, then CA4, 2,4,6-trichlorobenzoyl chloride, DMAP and triethylamine were dissolved in anhydrous DMF and added to the above mixture. The reaction was allowed to proceed at room temperature for $2 \mathrm{~h}$. After that, the reaction mixture was precipitated into excess diethyl ether, re-dissolved in DMF, and dialyzed against distilled water (MWCO 3500). The final product poly(L-aspartic acid)graft-methoxy poly(ethylene glycol)/combretastatin A4 


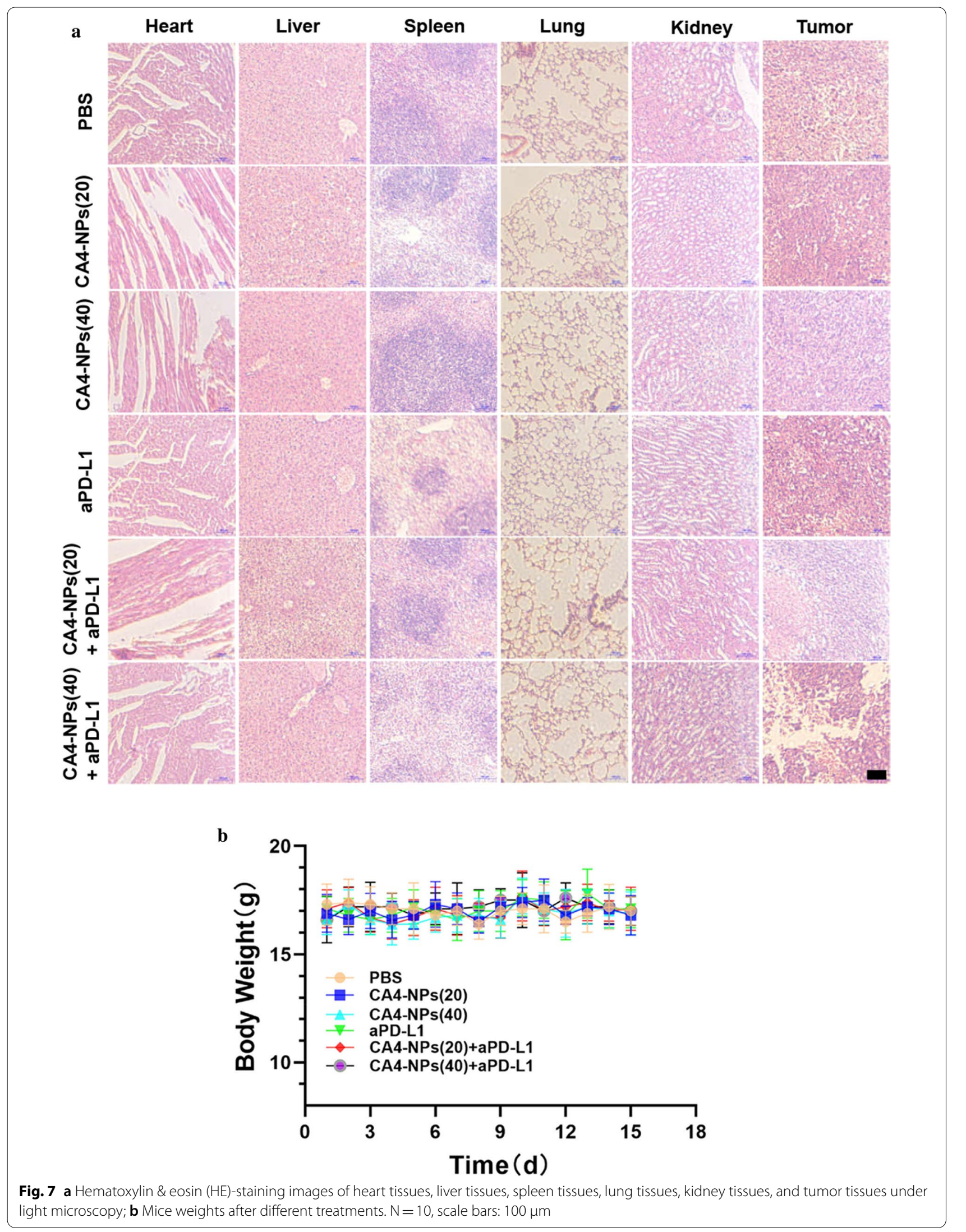


(PEG-b-PAsp-g-CA4) was obtained after lyophilization (Fig. 8).

\section{Characterization}

${ }^{1} \mathrm{H}$ NMR spectra of the polymers were recorded on a Bruker $400 \mathrm{MHz}$ nuclear magnetic resonance instrument using DMSO as the solvents. Gel permeation chromatography (GPC) was used to analyze the molecular weights and molecular weight distributions $(\mathrm{Mw} / \mathrm{Mn})$ of the polymers. GPC of PEG-b-PAsp was measured at room temperature with a Waters 1525 chromatograph equipped with a Waters 2414 refractive index detector. $\mathrm{H}_{2} \mathrm{O}$ was used as eluents with a low rate of $1.0 \mathrm{~mL} / \mathrm{min}$ and narrowly distributed polyethyleneglycol was used as standard. The size and surface charge of the nanocarrier was investigated on Malvern ZetasizerNano ZS 90 zeta potential analyzer. Ultraviolet-visible (UV-vis) spectra were collected using a LAMBDA-35 spectrometer. CA4 loading content was calculated by measuring the UV-Vis absorbance at $290 \mathrm{~nm}$.

Transmission electron microscopy (TEM) was performed on a JEOL-2100 with an accelerating voltage of
$200 \mathrm{kV}$. TEM samples were prepared by drop-casting dispersion onto copper grids covered by carbon film. Confocal images were acquired using a Zeiss confocal laser scanning unit mounted on an LSM 710 fixed-stage upright microscope (CLSM).

\section{In vitro release profiles}

In vitro release of CA4 from PLG-CA4 was conducted using a dialysis method. Briefly, 3.0 mg PLG-CA4 was dissolved in $10 \mathrm{~mL}$ distilled water, PBS solution at $\mathrm{pH}$ 7.4 or 5.5, and bovine albumin (BSA), respectively. Samples were sealed in a dialysis bag (MWCO $3500 \mathrm{Da})$ and incubated in $45 \mathrm{~mL}$ of PBS at $37^{\circ} \mathrm{C}$ with a shaking rate of $100 \mathrm{rpm}$. At predetermined time points $(1,2,4$, $8,12,24,36,48 \mathrm{~h}), 3 \mathrm{~mL}$ of the incubated solution was taken out and replaced with fresh media. The concentration of $\mathrm{CA} 4$ in the released media was determined by HPLC at $290 \mathrm{~nm}$ with a mobile phase of acetonitrile and water $(80 / 20, v / v)$, as described above.<smiles>CC(C)(C)[C@H](N)C(=O)OCc1ccccc1COC(=O)CC1NC(=O)OC1=O</smiles>

BLA-NCA

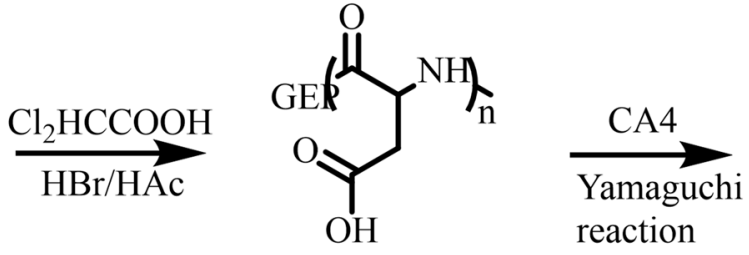

PEG-b-PAsp<smiles></smiles>

PEG-b-PAsp-p-CA4

Fig. 8 Synthesis procedures of PEG-b-PAsp-g-CA4 


\section{Critical micelle concentration (CMC)}

The CMC was determined using a Malvern Zetasizer, NANO ZS90 (Malvern Instruments Limited, U.K.) equipped with a laser light scattering spectrometer at $632.8 \mathrm{~nm}$ under room temperature $\left(25^{\circ} \mathrm{C}\right)$. Solutions containing different concentrations of PEG-b-PAsp and PEG-b-PAsp-g-CA4 were tested, and the light scattering intensity was recorded for each concentration analyzed.

\section{Cytotoxicity}

The cytotoxicity was analyzed by the MTT method. Primary hepatocytes were isolated and cultured according to a previous method and with the modification of our team [39]. Hepa1-6 cells, HepG2 cells, and LO2 cells were cultured in DMEM medium with 10\% FBS and primary hepatocytes were cultured in William's Medium E (supplemented with $5 \%$ fetal bovine serum, $100 \mathrm{U} / \mathrm{ml}$ penicillin, $100 \mu \mathrm{g} \mathrm{ml}^{-1}$ streptomycin, $1 \times$ ITS and $5 \mathrm{nM}$ dexamethasone) at $37{ }^{\circ} \mathrm{C}$ under $5 \% \mathrm{CO}_{2}$. Briefly, Hepa1-6 cells, HepG2 cells, LO2 cells and primary hepatocytes were seeded in 96-well plates at a density of $1 \times 10^{5}$ cell per well and cultured at $37{ }^{\circ} \mathrm{C}$. CA4, CA4P and PEG-bPAsp-g-CA4 with increasing $\mathrm{w} / \mathrm{w}$ ratio were added, after incubation for $48 \mathrm{~h}, 20 \mu \mathrm{L}$ MTT solution $\left(5 \mathrm{mg} \mathrm{mL}^{-1}\right)$ was added to each well. After incubation for $4 \mathrm{~h}, 100 \mu \mathrm{L}$ dimethyl sulfoxide was added to replace the media to dissolve formazan, the absorbance was analyzed at a wavelength of $490 \mathrm{~nm}$ with a microplate reader.

\section{Cellular uptake and intracellular trafficking}

Hepa1-6 cells were seeded into 24-well plates and then were incubated with the polyplexes prepared with FITC $\left(10,20,30\right.$, and $\left.40 \mu \mathrm{g} \mathrm{mL}^{-1}\right)$ and PEG-b-PAsp-g-CA4FITC (on the FITC basis) for 2,4 and $6 \mathrm{~h}$. The cells were then trypsinized and uptake-analyzed by the MACSQuant flow cytometer. Intracellular distribution was assessed by confocal microscopy following DAPI staining $4 \mathrm{~h}$ after incubation.

\section{Pharmacokinetics}

Pharmacokinetics was characterized by measuring CA4 levels by collecting the eye arterial blood of SD female rats (about $250 \mathrm{~g}$ ). Specifically, six rats were divided into two groups of 3 each, and they were injected with free CA4P (50.0 mg kg-1 on the basis of CA4) or PEG-b-PAspg-CA4 (50.0 mg kg ${ }^{-1}$ based on CA4) via tail veins, respectively. Eye arterial blood was collected at predetermined times $(2,5,15,30,60,90,120,240,360,480 \mathrm{~min})$ and was heparinized and centrifuged to obtain plasma. Next, $50 \mu \mathrm{L}$ of the plasma sample was deproteinized with $250 \mu \mathrm{L}$ of acetonitrile [contains $\alpha$-naphthylamine $\left(40 \mu \mathrm{g} \mathrm{mL}^{-1}\right)$ ], vortexed for $5 \mathrm{~min}$, and centrifuged at 10,000 rpm for $10 \mathrm{~min}$. The supernatant was filtered by using $0.22 \mu \mathrm{m}$ filters before HPLC measurement. The pharmacokinetics assay of PEG-b-PAsp-g-CA4 and CA4P was performed by fluorescence detector HPLC (FLD-HPLC) with a fluorescence detector at $290 \mathrm{~nm} E x$ and $380 \mathrm{~nm} \mathrm{Em}$, and the elution was performed with methanol and water $(\mathrm{v} / \mathrm{v}=7$ : 3) pumped at a flow velocity of $1.0 \mathrm{~mL} \mathrm{~min}^{-1}$ at $40^{\circ} \mathrm{C}$.

\section{In vivo imaging}

To explore the tumor-targeting capability of CA4-NPs in vivo, Hepa1-6-bearing mice were i.v. injected with Cy5.5-loaded nano drugs followed by near-infrared optical imaging. Briefly, mice were subcutaneously inoculated with Hepa1-6 cells $\left(1 \times 10^{7}\right.$ cells $)$ in the right flank. When tumor reached $150-200 \mathrm{~mm}^{3}$, mice were randomly divided into two groups (free Cy5.5 and PEG-bPAsp-g-CA4-Cy5.5) at an identical $50 \mu \mathrm{g} \mathrm{kg}{ }^{-1}$ Cy5.5. 2,6, 12 and $24 \mathrm{~h}$ after injection, NIR fluorescent images were captured by in vivo imaging system (Tanon ABL X6, China). Finally, mice were sacrificed and heart, liver, spleen, lung, kidneys and tumor of each mouse were harvested for more detailed comparison.

\section{Tissue biodistribution study}

To explore the tissue biodistribution study further, mice were subcutaneously inoculated with Hepa1-6 cells $\left(1 \times 10^{7}\right.$ cells $)$ in the right flank. When tumor reached $150-200 \mathrm{~mm}^{3}$, mice were randomly divided into two groups (free CA4P and PEG-b-PAsp-g-CA4) via the tail vein at dose of $40 \mathrm{mg} \mathrm{CA} 4 / \mathrm{kg}$. At $24 \mathrm{~h}$, the mice were killed and their tumors, hearts, livers, spleens, lungs, kidneys were collected. Tissue samples were washed in icecold saline blotted with paper towels to remove excess fluid and weighed. Then the tumors and main organs were homogenized in water $(1 \mathrm{~g}$ tissue per $1 \mathrm{~mL})$ to get suspensions. And $100 \mu \mathrm{L}$ tissue samples were deproteinized into $300 \mu \mathrm{L}$ of acetonitrile by vortex for $5 \mathrm{~min}$, and centrifuged at $10,000 \mathrm{rpm}$ for $10 \mathrm{~min}$. The test conditions of tissue biodistribution study was performed the same as pharmacokinetics assay.

\section{Immunofluorescence staining assay}

The expressions of CD31, HIF-1 $\alpha$, PD-L1, CD4, CD8, LAG-3, TIM-3 were analyzed by immunofluorescence staining assay. The tumor tissues were excised and fixed in $4 \%$ buffered paraformaldehyde for $24 \mathrm{~h}$, then embedded in paraffin and sliced to a thickness of $5.0 \mu \mathrm{m}$. The tumor tissue slides were deparaffinized by xylene, hydrated through a gradient of alcohol, boiled in $0.01 \mathrm{M}$ sodium citrate buffer ( $\mathrm{pH}$ 6.0) for antigen retrieval. After treating in blocking medium (5\% BSA PBS buffer) for $20 \mathrm{~min}$, the tumor tissue slices were first incubated with a monoclonal primary antibody (CD31, HIF-1 $\alpha$, PD-L1 CD4, CD8, LAG-3, TIM-3), and incubated with 
a fluorescently labeled secondary antibody (Cy3/AF488/ AF594), then imaged by confocal laser scanning microscopy (CLSM).

\section{Tumor models}

Healthy female C57BL/6 mice ( $20 \pm 2$ g) were purchased from Charles River (Beijing), and handling procedures were according to the guidelines of the Regional Ethics Committee for Animal Experiments of China Pharmaceutical University. Hepa1-6 tumor-bearing mice were selected as the animal model to assess the anti-tumor effect. Hepa1-6 cells were harvested after cell culture. Then, cells were suspended in PBS $\left(5 \times 10^{7}\right.$ cells $\mathrm{mL}^{-1}$, $200 \mu \mathrm{L}$ ) and subcutaneously injected near the mammary region of mice.

\section{In vivo antitumor efficacy}

When the tumor volume reached about $100 \mathrm{~mm}^{3}$, the nude mice were randomly divided into 6 groups with 6 mice in one group. Then the mice were administrated with PBS (group 1), CA4-NPs (20 mg kg-1) (group 2), CA4-NPs (40 mg kg $\mathrm{g}^{-1}$ (group 3), aPD-L1 (group 4), CA4-NPs $\left(20 \mathrm{mg} \mathrm{kg}^{-1}\right)+$ aPD-L1 (group 5), and CA4NPs (40 mg kg-1)+aPD-L1(group 6). The ratio of CA4 amount and body weight was about $5 \mathrm{mg} \mathrm{kg}^{-1}$ or $10 \mathrm{mg} \mathrm{kg}$. The dosage of aPD-L1 was $50 \mu \mathrm{L}$ for each mouse. The tumor size and body weight were recorded every day during the treatment. The mice were sacrificed after 15 days, and then the tumors and major organs were isolated and stained by hematoxylin \& eosin (H\&E). Besides, Ki67 and TUNEL immunostaining were also applied for all the tumors. Finally, the survival time of the remaining mice for each group were monitored additionally until day 60 and the survival curves were produced.

\section{Measurements of cytokines}

To evaluate the level of IFN- $\gamma$ and IL-4, frozen tumors were homogenized in PBS solution at $4{ }^{\circ} \mathrm{C}$. All homogenized samples were centrifuged and the supernatants were stored at $-80{ }^{\circ} \mathrm{C}$ before analysis. Meanwhile, the blood samples were centrifuged to obtain the serum. Then cytokines both in mice serum and tumor were analyzed with corresponding ELISA kits (R\&D Systems, Minneapolis, Minnesota, USA) according to the manufacturers' instruction.

\section{Statistical analysis}

Data were calculated and processed as mean \pm SD. Comparison between groups was analyzed with Student's t-test or one-way analysis of variance (ANOVA). Statistical differences were considered significant at * $\mathrm{p}<0.05$, ${ }^{* * *} \mathrm{p}<0.01,{ }^{* * * * *} \mathrm{p}<0.001,{ }^{* * * * * *} \mathrm{p}<0.001$.

\section{Supplementary Information}

The online version contains supplementary material available at https://doi. org/10.1186/s12951-021-00865-w.

Additional file 1: Figure S1. Images of the excised tumors after different treatments; shown are PBS-treated mice and mice receiving after injection with CA4-NPs (20 mg kg-1), CA4-NPs (40 $\left.\mathrm{mg} \mathrm{kg}^{-1}\right)$, aPD-L1, CA4-NPs (20 mg $\left.\mathrm{kg}^{-1}\right)+$ aPD-L1, CA4-NPs $\left(40 \mathrm{mg} \mathrm{kg}^{-1}\right)+$ aPD-L1, CA4P $\left(40 \mathrm{mg} \mathrm{kg}^{-1}\right), \mathrm{n}=10$. Figure S2 Tissue distributions of CA4 in mice receiving free CA4P and PEG-b-PAsp-g-CA4 at $24 \mathrm{~h}$. Figure $\mathbf{S} 3{ }^{1} \mathrm{H}$ NMR spectra of BLA-NCA.

\section{Acknowledgements}

Not applicable.

\section{Authors' contributions}

$\mathrm{BZ}, \mathrm{HH}$ and $\mathrm{YW}$ performed materials preparation and wrote the manuscript. $Z D$ and BZ performed the conception and design of the study. WL performed in vivo experiments. BZ performed in vitro experiments. FL and QW performed image analyses. All authors read and approved the final manuscript.

\section{Funding}

The authors gratefully acknowledge the support of Natural Science Foundation of Jiangsu Province (Project BK20201333) and "Double First-Class" University project (CPU2018GY25).

\section{Availability of data and materials}

All data generated or analyzed during this study are included in this published article.

\section{Declarations}

\section{Ethics approval and consent to participate}

All animal procedures were performed in accordance with the Guidelines for Care and Use of Laboratory Animals of China Pharmaceutical University and approved by the Animal Ethics Committee of China Pharmaceutical University, Jiangsu, China.

\section{Consent for publication}

Not applicable.

\section{Competing interests}

The authors declare that they have no competing interests.

Received: 30 December 2020 Accepted: 19 April 2021

Published online: 01 May 2021

References

1. Nelson ME, Lahiri S, Chow JD, Byrne FL, Hargett SR, Breen DS, et al. Inhibition of hepatic lipogenesis enhances liver tumorigenesis by increasing antioxidant defence and promoting cell survival. Nat Commun. 2017;8:14689.

2. Zeng H, Zheng R, Guo Y, Zhang S, Zou X, Wang N, et al. Cancer survival in China, 2003-2005: a population-based study. Int J Cancer. 2015;136(8):1921-30.

3. Forner A, Reig M, Bruix J. Hepatocellular carcinoma. Lancet. 2018;391(10127):1301-14.

4. Yan H, Chen J, Li Y, Bai Y, Wu Y, Sheng Z, et al. Ultrasmall hybrid proteincopper sulfide nanoparticles for targeted photoacoustic imaging of orthotopic hepatocellular carcinoma with a high signal-to-noise ratio. Biomater Sci. 2018;7(1):92-103.

5. de Baere T, Arai Y, Lencioni R, Geschwind JF, Rilling W, Salem R, et al. Treatment of liver tumors with lipiodol tace: technical recommendations from experts opinion. Cardiovasc Intervent Radiol. 2016;39(3):334-43.

6. Zhang ZH, Zhang W, Gu JY, Liu QX, Ma JQ, Liu LX, et al. Treatment of hepatocellular carcinoma with tumor thrombus with the use of iodine-125 
seed strand implantation and transarterial chemoembolization: a propensity-score analysis. J Vasc Interv Radiol. 2018;29(8):1085-93.

7. Chan SL, Yeo W, Mo F, Chan AWH, Koh J, Li L, et al. A phase 2 study of the efficacy and biomarker on the combination of transarterial chemoembolization and axitinib in the treatment of inoperable hepatocellular carcinoma. Cancer. 2017;123(20):3977-85.

8. Mao D, Zhu M, Zhang X, Ma R, Yang X, Ke T, et al. A macroporous heparin-releasing silk fibroin scaffold improves islet transplantation outcome by promoting islet revascularisation and survival. Acta Biomater. 2017:59:210-20

9. Shenoi MM, Iltis I, Choi J, Koonce NA, Metzger GJ, Griffin RJ, et al. Nanoparticle delivered vascular disrupting agents (VDAs): use of TNF-alpha conjugated gold nanoparticles for multimodal cancer therapy. Mol Pharm. 2013;10(5):1683-94.

10. Levy A, Albiges-Sauvin L, Massard C, Izzedine H, Ederhy S, Bahleda R, et al. Reappraisal of treatment-induced renal dysfunction in patients receiving antiangiogenic agents in phase I trials. Invest New Drugs. 2012;30(3):1116-20.

11. Close A. Antiangiogenesis and vascular disrupting agents in cancer: circumventing resistance and augmenting their therapeutic utility. Future Med Chem. 2016;8(4):443-62.

12. Chen $\mathrm{Q}$, Liu G, Liu S, Su H, Wang Y, Li J, et al. Remodeling the tumor microenvironment with emerging nanotherapeutics. Trends Pharmacol Sci. 2018:39(1):59-74.

13. Yu H, Tang Z, Zhang D, Song W, Duan T, Gu J, et al. Poly(ornithine-coarginine-co-glycine-co-aspartic acid): preparation via NCA polymerization and its potential as a polymeric tumor-penetrating agent. Macromol Biosci. 2015;15(6):829-38.

14. Wang Y, Yu H, Zhang D, Wang G, Song W, Liu Y, et al. Co-administration of combretastatin A4 nanoparticles and sorafenib for systemic therapy of hepatocellular carcinoma. Acta Biomater. 2019;92:229-40.

15. Jiang J, Shen N, Song W, Yu H, Sakurai K, Tang Z, et al. Combretastatin A4 nanodrug combined plerixafor for inhibiting tumor growth and metastasis simultaneously. Biomater Sci. 2019;7(12):5283-91.

16. Liu Z, Shen N, Tang Z, Zhang D, Ma L, Yang C, et al. An eximious and affordable GSH stimulus-responsive poly(alpha-lipoic acid) nanocarrier bonding combretastatin A4 for tumor therapy. Biomater Sci. 2019;7(7):2803-11.

17. Cao Y, Wei Z, Li M, Wang H, Yin L, Chen D, et al. Formulation, pharmacokinetic evaluation and cytotoxicity of an enhanced- penetration paclitaxel nanosuspension. Curr Cancer Drug Targets. 2019;19(4):338-47.

18. Wang QY, Xu YS, Zhang NX, Dong ZP, Zhao BN, Liu LC, et al. Phenylboronic ester-modified anionic micelles for ROS-stimuli response in HeLa cell. Drug Deliv. 2020;27(1):681-90.

19. Patra JK, Das G, Fraceto LF, Campos EVR, Rodriguez-Torres MDP, AcostaTorres LS, et al. Nano based drug delivery systems: recent developments and future prospects. J Nanobiotechnology. 2018;16(1):71.

20. Hargadon KM, Johnson CE, Williams CJ. Immune checkpoint blockade therapy for cancer: an overview of FDA-approved immune checkpoint inhibitors. Int Immunopharmacol. 2018;62:29-39.

21. Li B, Chan HL, Chen P. Immune checkpoint inhibitors: basics and challenges. Curr Med Chem. 2019;26(17):3009-25.

22. Wei SC, Duffy CR, Allison JP. Fundamental mechanisms of immune checkpoint blockade therapy. Cancer Discov. 2018;8(9):1069-86.

23. Hamanishi J, Mandai M, Matsumura N, Abiko K, Baba T, Konishi I. PD-1/ PD-L1 blockade in cancer treatment: perspectives and issues. Int J Clin Oncol. 2016;21(3):462-73.
24. Kuol N, Stojanovska L, Nurgali K, Apostolopoulos V. PD-1/PD-L1 in disease. Immunotherapy. 2018;10(2):149-60.

25. Ramjiawan RR, Griffioen AW, Duda DG. Anti-angiogenesis for cancer revisited: is there a role for combinations with immunotherapy? Angiogenesis. 2017;20(2):185-204

26. Noman MZ, Desantis G, Janji B, Hasmim M, Karray S, Dessen P, et al. PD-L1 is a novel direct target of HIF-1alpha, and its blockade under hypoxia enhanced MDSC-mediated T cell activation. J Exp Med. 2014:211(5):781-90

27. Dai X, Pi G, Yang SL, Chen GG, Liu LP, Dong HH. Association of PD-L1 and HIF-1 a coexpression with poor prognosis in hepatocellular carcinoma. Transl Oncol. 2018;11(2):559-66.

28. Zhao Y, Wang XX, Wu W, Long H, Huang J, Wang Z, et al. EZH2 regulates PD-L1 expression via HIF-1a in non-small cell lung cancer cells. Biochem Biophys Res Commun. 2019;517(2):201-9.

29. Chang YL, Yang CY, Lin MW, Wu CT, Yang PC. High co-expression of PD-L1 and $\mathrm{HIF-1a}$ correlates with tumour necrosis in pulmonary pleomorphic carcinoma. Eur J Cancer. 2016:60:125-35.

30. Lorusso PM, Boerner SA, Hunsberger S. Clinical development of vascular disrupting agents: what lessons can we learn from ASA404? J Clin Oncol. 2011;29(22):2952-5

31. Madlambayan GJ, Meacham AM, Hosaka K, Mir S, Jorgensen M, Scott EW, et al. Leukemia regression by vascular disruption and antiangiogenic therapy. Blood. 2010;1 16(9):1539-47.

32. Ho YJ, Wang TC, Fan CH, Yeh CK. Current progress in antivascular tumor therapy. Drug Discov Today. 2017;22(10):1503-15.

33. Jiang J, Shen N, Ci T, Tang Z, Gu Z, Li G, et al. Combretastatin A4 nanodrug-induced MMP9 amplification boosts tumor-selective release of doxorubicin prodrug. Adv Mater. 2019;31(44):e1904278.

34. Upadhaya S, Neftelino ST, Hodge JP, Oliva C, Campbell JR, Yu JX. Combinations take centre stage in PD1/PDL1 inhibitor clinical trials. Nat Rev Drug Discov. 2020;20:168-9.

35. Akdis M, Aab A, Altunbulakli C, Azkur K, Costa RA, Crameri R, et al. Interleukins (from IL-1 to IL-38), interferons, transforming growth factor beta, and TNF-alpha: receptors, functions, and roles in diseases. J Allergy Clin Immunol. 2016;138(4):984-1010.

36. Mimura K, Kua LF, Xiao JF, Asuncion BR, Nakayama Y, Syn N, et al. Combined inhibition of PD-1/PD-L1, Lag-3, and Tim-3 axes augments antitumor immunity in gastric cancer-T cell coculture models. Gastric Cancer. 2021. https://doi.org/10.1007/s10120-020-01151-8.

37. Datar I, Sanmamed MF, Wang J, Henick BS, Choi J, Badri T, et al. Expression analysis and significance of PD-1, LAG-3, and TIM-3 in Human Non-small cell lung cancer using spatially resolved and multiparametric single-cell analysis. Clin Cancer Res. 2019;25(15):4663-73.

38. El Halabi L, Adam J, Gravelle P, Marty V, Danu A, Lazarovici J, et al. Expression of the immune checkpoint regulators LAG-3 and TIM-3 in classical Hodgkin lymphoma. Clin Lymphoma Myeloma Leuk. 2020;21:257-266.e3.

39. Zheng $X$, Zhao MG, Jiang $C H$, Sheng $X P$, Yang HM, Liu Y, et al. Triterpenic acids-enriched fraction from Cyclocarya paliurus attenuates insulin resistance and hepatic steatosis via PI3K/Akt/GSK3beta pathway. Phytomedicine. 2020;66:153130.

\section{Publisher's Note}

Springer Nature remains neutral with regard to jurisdictional claims in published maps and institutional affiliations. 